\title{
Convergence-Optimal Quantizer Design of Distributed Contraction-based Iterative Algorithms with Quantized Message Passing
}

\author{
Ying Cui, Student Member, IEEE, Vincent K. N. Lau, Senior Member, IEEE, \\ Department of ECE, The Hong Kong University of Science and Technology \\ cuiying@ust.hk,eeknlau@ee.ust.hk
}

\begin{abstract}
In this paper, we study the convergence behavior of distributed iterative algorithms with quantized message passing. We first introduce general iterative function evaluation algorithms for solving fixed point problems distributively. We then analyze the convergence of the distributed algorithms, e.g. Jacobi scheme and GaussSeidel scheme, under the quantized message passing. Based on the closed-form convergence performance derived, we propose two quantizer designs, namely the time invariant convergence-optimal quantizer (TICOQ) and the time varying convergence-optimal quantizer (TVCOQ), to minimize the effect of the quantization error on the convergence. We also study the tradeoff between the convergence error and message passing overhead for both TICOQ and TVCOQ. As an example, we apply the TICOQ and TVCOQ designs to the iterative waterfilling algorithm of MIMO interference game.
\end{abstract}

\section{INTRODUCTION}

Distributed algorithm design and analysis is a very important topic with important applications in many areas such as deterministic network utility maximization (NUM) for wireless networks and non-cooperative game. For example, in [1], [2], the authors derived various distributed algorithms for a generic deterministic NUM problem using the decomposition techniques, which can be classified into primal decomposition and dual decomposition methods. In [3], the authors investigated a distributed power control algorithm for an interference channel using non-cooperative game and derived an iterative water-filling algorithm to approach the Nash equilibrium (NE). The interference game problem was extended to iterative waterfilling algorithm for a wideband interference game with time/frequency offset in [4] and an iterative precoder optimization algorithm for a MIMO interference game in [5], [6]. The authors established a unified convergence proof of the iterative water-filling algorithms for the SISO frequencyselective interference game and the MIMO interference game using a contraction mapping approach. Using this framework, the iterative best response update (such as the iterative power water-filling as well as the iterative precoder design) can be regarded as an iterative function evaluations w.r.t. a certain contraction mapping and the convergence property can be easily established using fixed point theory [7], [8]. In all these examples, the iterative function evaluation algorithms involved explicit message passing between nodes in the wireless networks during the iteration process. Furthermore, these existing results have assumed perfect message passing during the iterations.

In practice, explicit message passing during the iterations in the distributed algorithms requires explicit signaling in wireless networks. As such, the message passing cannot be perfect and in many cases, the messages to pass have to be quantized. As a result, it is very important and interesting to study about the impact of quantized message passing on the convergence of the distributed algorithms. Existing studies on the distributed algorithms under quantized message passing can be classified into two categories, namely the distributed quantized average consensus algorithms [9][14] as well as the distributed quantized incremental subgradient algorithms [15]-[18]. For the distributed quantized average consensus algorithms, existing works considered the algorithm convergence performance under quantized message passing for uniform quantizer [9], [10], [12]-[14] and logarithmic quantizer [11] with fixed quantization rate. In [12], [14], the authors also considered quantization interval optimization (for average consensus algorithms) based on the uniform fixed-rate quantization structure. Similarly, for the second category of quantized incremental subgradient algorithms, the authors in [15]-[18] considered the convergence performance of fixed-rate uniform quantization. In this paper, we are interested in the convergence behavior of distributed iterative algorithms for solving general fixed point problems under quantized message passing. The above works on quantized message passing cannot be applied to our case due to the following reasons. First of all, the algorithm dynamics of the existing works (linear dynamics for average consensus algorthms and step-size 
based algorithms for incremental subgradient algorithms) are very different from the contraction-based iterative algorithms we are interested in (for solving fixed point problems). Secondly, the above works have imposed simplifying constraints of uniform and fixed rate quantizer design and it is not known if a more general quantizer design or adaptive quantization rate could further improve the convergence performance of the iterative algorithms. There are a few technical challenges regarding the study of convergence behavior in distributed contraction-based iterative function evaluations.

- Convergence Analysis and Performance Tradeoff under Quantized Message Passing: In the literature, convergence of distributed iterative function evaluation algorithms under quantized message passing has not been considered. The general model under quantized message passing and how does the quantization error affect the convergence are not fully studied. Furthermore, it will also be interesting to study the tradeoff between convergence error and message passing overhead.

- Quantizer Design based on the Convergence Performance: Given the convergence analysis results, how to optimize the quantizer to minimize the effect of the quantization error on the convergence is a difficult problem. In general, quantizers are designed w.r.t. a certain distortion measure such as the mean square error [19], [20]. However, it is not clear which distortion measure we should use to design the quantizer in order to optimize the convergence performance of the iterative algorithms we considered. Furthermore, the convergence performance highly depends on the quantizer structure as well as the quantization rate, and hence, a lowcomplexity solution to the nonlinear integer quantizer optimization problem is of great importance.

In this paper, we shall attempt to shed some lights on these questions. We shall first introduce a general iterative function evaluation algorithm with distributed message passing for solving fixed point problems. We shall then analyze the convergence of the distributed algorithms, e.g. Jacobi scheme and Gauss-Seidel scheme, under the quantized message passing. Based on the analysis, we shall propose two rate-adaptive quantizer designs, namely the time invariant convergence-optimal quantizer (TICOQ) and the time varying convergenceoptimal quantizer (TVCOQ), to minimize the effect of the quantization error on the convergence. We shall also develop efficient algorithms to solve the nonlinear integer programming problem associated with the quantizer optimization problem. As an illustrative example, we shall apply the TICOQ and TVCOQ designs to the iterative waterfilling algorithm of the MIMO interference game [5], [6].
We first list the important notations in this paper in table I]

\begin{tabular}{|c|c|}
\hline$n$ & dimension of vector of state variables \\
$m(1 \leq m \leq n)$ & element index of vector \\
number of nodes/blocks \\
$k(1 \leq k \leq K)$ & node index/block index \\
$\bar{T}$ & total number of iterations \\
$t(1 \leq t \leq \bar{T})$ & iteration index \\
$\mathcal{Q}_{k}$ & component quantizer of node $k$ (general) \\
$\mathcal{Q}=\left(\mathcal{Q}_{1}, \cdots, \mathcal{Q}_{K}\right)$ & system quantizer (general) \\
superscript $s$ & scalar quantizer (SQ) \\
superscript $v$ & vector quantizer (VQ) \\
$\mathcal{Q}_{k}^{s}=\left(\mathcal{Q}_{m}^{s}\right)_{m \in \mathcal{M}_{k}}$ & component quantizer of node $k$ (SQ) \\
$\mathcal{Q}^{s}=\left(\mathcal{Q}_{1}^{s}, \cdots, \mathcal{Q}_{n}^{s}\right)$ & system quantizer (SQ) \\
$\mathbf{I}^{s}=\left(I_{1}^{s}, \cdots, I_{n}^{s}\right)$ & quantization index vector (SQ) \\
$\mathbf{L}^{s}=\left(L_{1}^{s}, \cdots, L_{n}^{s}\right)$ & quantization rate vector (SQ) \\
$\mathcal{Q}_{k}^{v}$ & component quantizer of node $k$ (VQ) \\
$\mathcal{Q}^{v}=\left(\mathcal{Q}_{1}^{v}, \cdots, \mathcal{Q}_{K}^{v}\right)$ & system quantizer (VQ) \\
$\mathbf{I}^{v}=\left(I_{1}^{v}, \cdots, I_{K}^{v}\right)$ & quantization index vector (VQ) \\
$\mathbf{L}^{v}=\left(L_{1}^{v}, \cdots, L_{K}^{v}\right)$ & quantization rate vector (VQ) \\
$\mathbb{R}^{+}$ & the set of nonnegative real numbers \\
$\mathbb{Z}^{+}$ & the set of nonnegative integers \\
\hline
\end{tabular}

TABLE I

LIST OF IMPORTANT NOTATIONS.

\section{ITERATIVE FunCtion EVAluATIONS}

In this section, we shall introduce the basic iterative function evaluation algorithm to solve fixed point problems as well as its parallel and distributed implementations. We shall then review the convergence property under perfect message passing in the iteration process. We shall also illustrate the application of the framework using the MIMO interference game in [5], [6] as an example.

\section{A. A General Framework of Iterative Function Evalua- tion Algorithms}

In algorithm designs of wireless systems, many iterative algorithms can be described as the following dynamic update equation [7]:

$$
\mathbf{x}(t+1)=\mathbf{T}(\mathbf{x}(t))
$$

where $\mathbf{x}(t) \in \mathbb{R}^{n}$ is the vector of state variables of the system at (discrete) time $t$ and $\mathbf{T}$ is a mapping from a subset $\mathcal{X} \subseteq \mathbb{R}^{n}$ into itself. Such iterative algorithm with dynamics described by (1) is called the iterative function evaluation algorithm, which is widely used to solve fixed point problems [7], [8]. Specifically, any vector $\mathrm{x}^{*} \in \mathcal{X}$ satisfying $\mathbf{T}\left(\mathbf{x}^{*}\right)=\mathbf{x}^{*}$ is called a fixed point of $\mathbf{T}$. If the sequence $\{\mathbf{x}(t)\}$ converges to some $\mathbf{x}^{*} \in \mathcal{X}$ and $\mathbf{T}$ is continuous at $\mathbf{x}^{*}$, then $\mathbf{x}^{*}$ is a fixed point of $\mathbf{T}$ [7]. Therefore, the iteration in (11) can be viewed as an algorithm for finding such a fixed point of $\mathbf{T}$. We shall first review a few properties below related to the convergence of (1D. Specifically, $\mathbf{T}$ is called a contraction 
mapping if it satisfies some property, which is defined as follows:

Definition 1 (Contraction Mapping): Let $\mathbf{T}: \mathcal{X} \rightarrow$ $\mathcal{X}$ be a mapping from a subset $\mathcal{X} \subseteq \mathbb{R}^{n}$ into itself satisfying the following property $\|\mathbf{T}(\mathbf{x})-\mathbf{T}(\mathbf{y})\| \leq$ $\alpha\|\mathbf{x}-\mathbf{y}\|(\forall \mathbf{x}, \mathbf{y} \in \mathcal{X})$, where $\|\cdot\|$ is some norm and $\alpha \in[0,1)$ is a constant scalar. Then the mapping $\mathbf{T}$ is called a contraction mapping and the scalar $\alpha$ is called the modulus of $\mathbf{T}$.

Remark 1: (Comparison with Step-size Based Incremental Subgradient Algorithms) The incremental subgradient algorithms [21] can be described as $\mathbf{x}(t+1)=$ $\mathbf{x}(t)-\epsilon_{t} g(\mathbf{x}(t))$, where $\left\{\epsilon_{t}\right\}$ is the step-size sequence and $g(\mathbf{x}(t))$ is a subgradient of the objective function at $\mathbf{x}(t)$ in a minimization problem. Such step-size based update algorithms and their associated convergence dynamics are quite different from the iterative function evaluation algorithm we considered in (1).

If $\mathbf{T}$ is a contraction mapping, then the iterative update in (1) is called contracting iteration. The convergence of (11) is summarized as follows (Proof can be found in [7]):

Theorem 1 (Convergence of Contracting Iterations): Suppose that $\mathbf{T}: \mathcal{X} \rightarrow \mathcal{X}$ is a contraction mapping with modulus $\alpha \in[0,1)$ and that $\mathcal{X} \subseteq \mathbb{R}^{n}$ is closed. We have:

(1) (Existence and Uniqueness of Fixed Points) The mapping $\mathbf{T}$ has a unique fixed point $\mathrm{x}^{*} \in \mathcal{X}$.

(2) (Geometric Convergence) For any initial vector $\mathbf{x}(0) \in \mathcal{X}$, the sequence $\{\mathbf{x}(t)\}$ generated by (1) converges to $\mathbf{x}^{*}$ geometrically. In particular, $\left\|\mathbf{x}(t)-\mathbf{x}^{*}\right\| \leq$ $\alpha^{t}\left\|\mathbf{x}(0)-\mathbf{x}^{*}\right\| \quad \forall t \geq 0$.

In the above discussion, $\|\cdot\|$ can be any well-defined norm. There are many useful norms in the literature. However, the commonly used norms can be classified into two groups, namely weighted maximum norm and $L_{p}$ norm $(1 \leq p<\infty)$. They are elaborated below:

- Weighted maximum norm:

$$
\|\mathbf{x}\|_{\infty}^{\mathbf{a}}=\max _{m} \frac{\left|x_{m}\right|}{a_{m}}\left(a_{m}>0\right)
$$

Note that for $a_{m}=1 \forall m$, this reduces to the maximum norm, which can also be obtained from the $L_{p}$ norm by taking the limit $p \rightarrow \infty$.

- $L_{p}$ norm $(1 \leq p<\infty)$ :

$$
\|\mathbf{x}\|_{p}=\left(\sum_{m=1}^{n}\left|x_{m}\right|^{p}\right)^{\frac{1}{p}}
$$

Note that for $p=1$ we get the taxicab norm and for $p=2$ we get the Euclidean norm.

\section{B. Parallel and Distributed Implementation of Contract- ing Iterations}

In practice, large scale computation always involves a number of processors or communication nodes jointly executing a computational task. As a result, parallel and distributed implementation is of prime importance. Information acquisition and control are within geographically distributed nodes, in which distributed computation is more preferable. In this part, we shall discuss the efficient parallel and distributed computation of the contracting iteration in (1).

To perform efficient parallel and distributed implementations with $K$ processors, the set $\mathcal{X}$ is partitioned into a Cartesian product of lower dimensional sets, based on the computational complexity consideration or the local information extraction and control requirement. Mathematically, it can be expressed as $\mathcal{X}=\prod_{k=1}^{K} \mathcal{X}_{k}$, where $\mathcal{X}_{k} \subseteq \mathbb{R}^{n_{k}}$ and $\sum_{k=1}^{K} n_{k}=n$. Let $n_{0}=0$ and $\mathcal{M}_{k}=\left\{m \in \mathbb{N}: \sum_{l=1}^{k} n_{l-1}+1 \leq m \leq \sum_{l=1}^{k} n_{l}\right\}$ be the index set of the $k$-th component set $\mathcal{X}_{k}(1 \leq k \leq K)$, where $\mathbb{N}$ is the set of integers. Thus, $\mathcal{X}_{k}=\prod_{m \in \mathcal{M}_{k}} \mathcal{X}_{m}$, where $\mathcal{X}_{m} \subseteq \mathbb{R}^{1}$. Any vector $\mathbf{x} \in \mathcal{X}$ is decomposed as $\mathbf{x}=\left(\mathbf{x}_{1}, \cdots, \mathbf{x}_{K}\right)$ with the $k$-th block component $\mathbf{x}_{k}=\left(x_{m}\right)_{m \in \mathcal{M}_{k}} \in \mathcal{X}_{k}=\prod_{m \in \mathcal{M}_{k}} \mathcal{X}_{m}$ and the mapping $\mathbf{T}: \mathcal{X} \rightarrow \mathcal{X}$ is decomposed as $\mathbf{T}(\mathbf{x})=$ $\left(\mathbf{T}_{1}(\mathbf{x}), \cdots, \mathbf{T}_{K}(\mathbf{x})\right)$ with the $k$-th block component $\mathbf{T}_{k}: \mathcal{X} \rightarrow \mathcal{X}_{k}$

When the set $\mathcal{X}$ is a Cartesian product of lower dimensional sets $\mathcal{X}_{k}$, block-parallelization with $K$ processors can be implemented by assigning one processor to update a different block component. The most common updating strategies for $\mathbf{x}_{1}, \cdots, \mathbf{x}_{K}$ based on the block mapping $\mathbf{T}$ are:

- Jacobi Scheme: All block components $\mathbf{x}_{1}, \cdots, \mathbf{x}_{K}$ are updated simultaneously, i.e.

$$
\mathbf{x}_{k}(t+1)=\mathbf{T}_{k}(\mathbf{x}(t)), \quad 1 \leq k \leq K
$$

- Gauss-Seidel Scheme: All block components $\mathbf{x}_{1}, \cdots, \mathbf{x}_{K}$ are updated sequentially, one after the other, i.e.

$$
\mathbf{x}_{k}(t+1)=\mathbf{S}_{k}(\mathbf{x}(t)), \quad 1 \leq k \leq K
$$

where $\mathbf{S}_{k}: \mathcal{X} \rightarrow \mathcal{X}_{k}$ given by

$$
\begin{aligned}
& \mathbf{S}_{k}(\mathbf{x}) \\
= & \begin{cases}\mathbf{T}_{k}(\mathbf{x}), & k=1 \\
\mathbf{T}_{k}\left(\mathbf{S}_{1}(\mathbf{x}), \cdots, \mathbf{S}_{k-1}(\mathbf{x}), \mathbf{x}_{k}, \cdots, \mathbf{x}_{K}\right), & 2 \leq k \leq K\end{cases}
\end{aligned}
$$

is the $k$-th block component of the GuassSeidel mapping $\mathrm{S}: \mathcal{X} \rightarrow \mathcal{X}$, i.e. $\mathrm{S}(\mathbf{x})=$ $\left(\mathbf{S}_{1}(\mathbf{x}), \cdots, \mathbf{S}_{K}(\mathbf{x})\right)$.

Both Jacobi Scheme and Gauss-Seidel Scheme belong to synchronous update schemes. Specifically, Jacobi Scheme assumes the network is synchronized, while the

\footnotetext{
${ }^{1}$ Due to page limit, we shall illustrate the design for synchronous updates in (4) and 5. However, the scheme can be extended to deal with totally asynchronous updates easily [7], which will be further illustrated later in footnote 5
} 
Gauss-Seidel Scheme assumes the network provides a (Hamiltonian) cyclic route [7].

The general weighted block-maximum norm on $\mathbb{R}^{n}$, which is usually associated with the block partition of the vector $\mathbf{x}$, is defined as [7]:

$$
\|\mathbf{x}\|_{\text {block }}^{\mathbf{w}}=\max _{k} \frac{\left\|\mathbf{x}_{k}\right\|_{k}}{w_{k}}
$$

where $\mathbf{w}=\left(w_{1}, \cdots, w_{K}\right)>0$ is the vector weight and $\|\cdot\|_{k}$ is the norm for the $k$-th block componen ${ }^{2} \mathbf{x}_{k}$, which can be any given norm on $\mathbb{R}^{n_{k}}$, such as the weighted maximum norm and the $L_{p}$ norm $(1 \leq p<\infty)$ defined in (2) and (3). The mapping $\mathbf{T}: \mathcal{X} \rightarrow \mathcal{X}$ is called a blockcontraction with modulus $\alpha \in[0,1)$ if it is a contraction under the above induced weighted block-maximum norm $\|\cdot\|_{\text {block }}^{\mathrm{w}}$ with modulus $\alpha$. The convergence of the Jacobi scheme and Gauss-Seidel scheme based on the blockcontraction is summarized in the following theorem [7]:

Theorem 2: (Convergence of Jacobi Scheme and Gauss-Seidel Scheme) If $\mathbf{T}: \mathcal{X} \rightarrow \mathcal{X}$ is a blockcontraction, then the Gauss-Seidel mapping $\mathbf{S}$ is also a block-contraction with the same modulus as $\mathbf{T}$. Furthermore, if $\mathcal{X}$ is closed, then the sequence $\{\mathbf{x}(t)\}$ generated by both the Jocobi scheme in (4) and the Gauss-Seidel scheme in (5) based on the mapping $\mathbf{T}$ converges to the unique fixed point $\mathrm{x}^{*} \in \mathcal{X}$ of $\mathbf{T}$ geometrically.

\section{Application Example - MIMO Interference Game}

The contracting iteration in (1) is very useful in solving fixed point problems. Fixed point problem is highly related to distributed resource optimization problems in wireless systems [3], [5], [6], [22]. For example, finding the Nash Equilibrim (NE) of a game is a fixed point problem. In this subsection, we shall illustrate the application of contracting iterations using MIMO interference game [5], [6] as an example.

Consider a system with $K$ noncooperative transmitterreceiver pairs communicating simultaneously over a MIMO channel with $N$ transmit antenna and $N$ receive antenna [5], [6]. The received signal of the $k$-th receiver is given by:

$$
\mathbf{y}_{k}=\mathbf{H}_{k k} \mathbf{s}_{k}+\sum_{j \neq k} \mathbf{H}_{j k} \mathbf{s}_{j}+\mathbf{n}_{k}
$$

where $\mathbf{s}_{k} \in \mathbb{C}^{N}$ and $\mathbf{y}_{k} \in \mathbb{C}^{N}$ are the vector transmitted by the $k$-th transmitter and the vector received by the $k$-th receiver respectively, $\mathbf{H}_{k k} \in \mathbb{C}^{N \times N}$ is the direct-channel of the $k$-th link, $\mathbf{H}_{j k} \in \mathbb{C}^{N \times N}$ is the cross-channel from the $j$-th transmitter to the $k$-th receiver, and $\mathbf{n}_{k} \in \mathbb{C}^{N}$ is a zero-mean circularly symmetric complex Gaussian noise

\footnotetext{
${ }^{2}$ Since in general, the norm of each block component may not be the same, subscript $k$ is used in $\|\cdot\|_{k}$.
}

vector with covariance matrix $\mathbf{R}_{\mathbf{n}_{k}}$. For each transmitter $k$, the total average transmit power is given by

$$
\mathbb{E}\left[\left\|\mathbf{s}_{k}\right\|_{2}^{2}\right]=\operatorname{Tr}\left(\mathbf{P}_{k}\right) \leq P_{k}
$$

where $\operatorname{Tr}(\cdot)$ denotes the trace operator, $\mathbf{P}_{k} \triangleq \mathbb{E}\left[\mathbf{s}_{k} \mathbf{s}_{k}^{H}\right]$ is the covariance matrix of the transmitted vector $\mathbf{s}_{k}$ and $P_{k}$ is the maximum average transmitted power. The maximum throughput of link $k$ for a given set of users' covariance matrices $\mathbf{P}_{1}, \cdots, \mathbf{P}_{K}$ is as follows

$$
r_{k}\left(\mathbf{P}_{k}, \mathbf{P}_{-k}\right)=\log \operatorname{det}\left(\mathbf{I}+\mathbf{H}_{k k}^{H} \mathbf{R}_{-k}^{-1}\left(\mathbf{P}_{-k}\right) \mathbf{H}_{k k} \mathbf{P}_{k}\right)
$$

where $\mathbf{R}_{-k}\left(\mathbf{P}_{-k}\right) \triangleq \mathbf{R}_{\mathbf{n}_{k}}+\sum_{j \neq k} \mathbf{H}_{j k} \mathbf{P}_{j} \mathbf{H}_{j k}^{H}$ is the noise covariance matrix plus the MUI observed by user $k$, and $\mathbf{P}_{-k} \triangleq\left(\mathbf{P}_{j}\right)_{j \neq k}$ is the covariance matrix of all other users except user $k$.

In the MIMO interference game [5], [6], each player $k$ competes against the others by choosing his transmit covariance matrix $\mathbf{P}_{k}$ (i.e., his strategy) that maximizes his own maximum throughput $r_{k}\left(\mathbf{P}_{k}, \mathbf{P}_{-k}\right)$ in (10), subject to the transmit power constraint in (9), the mathematical structure of which is as follows

$$
\begin{aligned}
(\mathcal{G}) \max _{\mathbf{P}_{k}} & r_{k}\left(\mathbf{P}_{k}, \mathbf{P}_{-k}\right) \quad \forall k \\
\text { s.t. } & \mathbf{P}_{k} \in \mathscr{P}_{k}
\end{aligned}
$$

where $\mathscr{P}_{k} \triangleq\left\{\mathbf{P}_{k} \in \mathbb{C}^{N \times N}: \mathbf{P}_{k} \succcurlyeq 0, \operatorname{Tr}\left(\mathbf{P}_{k}\right)=P_{k}\right\}$ is the admissible strategy set of user $k$, and $\mathbf{P}_{k} \succcurlyeq$ 0 denotes that $\mathbf{P}_{k}$ is a positive semidefinite matrix. Given $k$ and $\mathbf{P}_{-k} \in \mathscr{P}_{-k}$, the solution to the noncooperative game (11) is the well-know waterfilling solution $\mathbf{P}_{k}^{*}=\mathbf{W F}_{k}\left(\mathbf{P}_{-k}\right)$, where the waterfilling operator $\mathbf{W F}_{k}\left(\mathbf{P}_{-k}\right)$ can be equivalently written as [5]

$$
\mathbf{W F}_{k}\left(\mathbf{P}_{-k}\right)=\left[-\left(\mathbf{H}_{k k}^{H} \mathbf{R}_{-k}^{-1}\left(\mathbf{P}_{-k}\right) \mathbf{H}_{k k}\right)^{-1}\right]_{\mathscr{P}_{k}}
$$

where $\left[\mathbf{X}_{0}\right]_{\mathscr{X}}=\arg \min _{\mathbf{Z} \in \mathscr{X}}\left\|\mathbf{Z}-\mathbf{X}_{0}\right\|_{F}$ denotes the matrix projection of $\mathbf{X}_{0}$ w.r.t Frobenius norm $\|\cdot\|_{F}$ onto the set $\mathscr{X}$. The NE of the MIMO Gaussian interference game is the fixed point solution of the waterfilling mapping WF $: \mathscr{P} \rightarrow \mathscr{P}$, i.e. $\mathbf{P}_{k}^{*}=\mathbf{W F}_{k}\left(\mathbf{P}_{-k}^{*}\right) \forall k$, where $\mathscr{P} \triangleq \mathscr{P}_{1} \times \cdots \times \mathscr{P}_{K}$ and $\mathbf{W F}=\left(\mathbf{W F}_{1}, \cdots, \mathbf{W F}_{K}\right)$.

In [5], it is shown that under some mild condition, the mapping WF is a block-contraction 4 w.r.t. $\|\cdot\|_{F, \text { block }}^{\mathbf{w}}$. Therefore, the NE can be achieved by the following contracting iteration

$$
\mathbf{P}(t+1)=\mathbf{W F}(\mathbf{P}(t))
$$

${ }^{3}$ If we arrange $M N$ elements of a $M \times N$ matrix $\mathbf{X}$ as a $M N$-dimensional vector $\mathbf{x}$, then the Frobenius norm of matrix $\mathbf{X}$ is equivalent to the $L_{2}$ norm of vector $\mathbf{x}$.

${ }^{4}$ After rearranging the elements of the $N \times N$ covariance matrix $\mathbf{P}_{k}$ as a $N^{2}$-dimensional vector, the block-contraction $\mathbf{W F}$ w.r.t. $\|\cdot\|_{F \text {,block }}^{\mathbf{w}}$ is equivalent to a block-contraction w.r.t. $\|\cdot\|_{\text {block }}^{\mathbf{w}}$ defined in (7) with each $\|\cdot\|_{k}$ being $L_{2}$ norm. 
where $\mathbf{P}=\left(\mathbf{P}_{1}, \cdots, \mathbf{P}_{K}\right)$. It can be easily seen that the waterfilling algorithm for the MIMO interference game in 13) is a special case of the contracting iterations in (1). In our general model, $\mathbf{x}$ in (1) corresponds to $\mathbf{P}$ in (13); the block-contraction mapping $\mathbf{T}$ in (1) corresponds to $\mathbf{W F}$ in 13); the $k$-th block component $\mathbf{x}_{k}$ corresponds to the covariance matrix $\mathbf{P}_{k}$; the $k$-th block component mapping $\mathbf{T}_{k}$ corresponds to $\mathbf{W} \mathbf{F}_{k}$.

For the parallel and distributed implementation, we can partition the variable space $\mathscr{P}=\prod_{k} \mathscr{P}_{k}$, where each variable space $\mathscr{P}_{k}$ corresponds to the covariance matrix of the $k$-th link. In each iteration, the receiver of each link $k$ needs to locally measure the PSD of the interference received from the transmitter of the other links, i.e. $\sum_{j \neq k} \mathbf{H}_{j k} \mathbf{P}_{j}(t) \mathbf{H}_{j k}^{H}$, computes the covariance matrix of the $k$-th link and transmits the computational results to the associated transmitter. There are two distributed iterative waterfilling algorithms (IWFA) based on this waterfilling block-contraction, namely simultaneous IWFA and sequential IWFA, which are described as follows:

- Simultaneous IWFA: It is an example of the Jacobi scheme, which is given by

$$
\mathbf{P}_{k}(t+1)=\mathbf{W F}\left(\mathbf{P}_{-k}(t)\right), 1 \leq k \leq K
$$

- Sequential IWFA: It is an example of the GaussSeidel scheme, which is given by

$\mathbf{P}_{k}(t+1)= \begin{cases}\mathbf{W F}\left(\mathbf{P}_{-k}(t)\right), & \text { if }(t+1) \bmod K=k \\ \mathbf{P}_{k}(t), & \text { otherwise }\end{cases}$

\section{CONTRACTING ITERATIONS UNDER QUANTIZED Message PASsing}

In this section, we shall study the impact of the quantized message passing on the contracting iterations. We shall first introduce a general quantized message passing model, followed by some general results regarding the convergence behavior under quantized message passing.

\section{A. General Model of Quantized Message Passing}

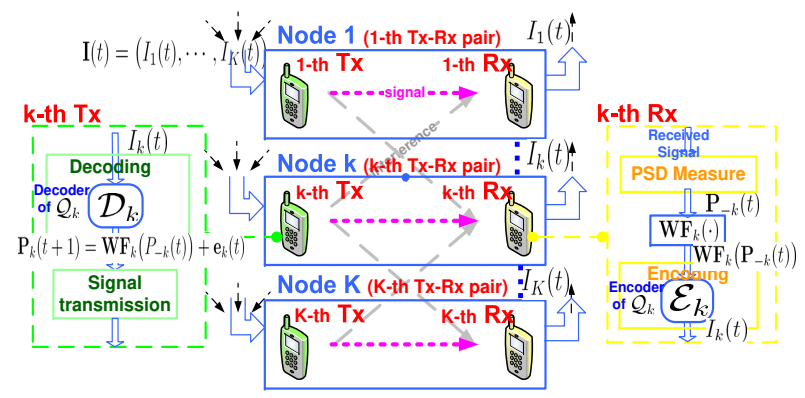

Fig. 1. Illustration of $K$-pair MIMO interference game.
We assume there are $K$ processing nodes geographically distributed in the wireless systems. Fig. 1 illustrates an example of $K$-pair MIMO interference game with quantized message passing. The system quantizer can be characterized by the tuple $\mathcal{Q}=\left(\mathcal{Q}_{1}, \ldots, \mathcal{Q}_{K}\right)$, where $\mathcal{Q}_{k}$ is the component quantizer (can be scalar or vector quantizer) for the $k$-th node. $\mathcal{Q}_{k}$ can be further denoted by the tuple $\mathcal{Q}_{k}=\left(\mathcal{E}_{k}, \mathcal{D}_{k}\right)$. $\mathcal{E}_{k}: \mathcal{X}_{k} \rightarrow \mathcal{I}_{k}$ is an encoder and $\mathcal{D}_{k}: \mathcal{I}_{k} \rightarrow \hat{\mathcal{X}}_{k}$ is a decoder. $\mathcal{I}_{k}=\left\{1, \cdots, 2^{L_{k}}\right\}$ and $L_{k}$ are the index set and the quantization rate of the component quantizer $\mathcal{Q}_{k} . \hat{\mathcal{X}}_{k}$ is the reproduction codebook, which is the set of all possible quantized outputs of $\mathcal{Q}_{k}$ [19]. The quantization rule is completely specified by $\mathcal{Q}_{k}: \mathcal{X}_{k} \rightarrow \hat{\mathcal{X}}_{k}$. Specifically, the quantized value is given by $\hat{\mathbf{x}}_{k}=\mathcal{Q}_{k}\left(\mathbf{x}_{k}\right)=\mathcal{D}_{k}\left(\mathcal{E}_{k}\left(\mathbf{x}_{k}\right)\right)$. Each node $k$ updates the $k$-th block component $\mathbf{x}_{k}$ of the $n$-dimensional vector $\mathbf{x}$, i.e. computes $\mathbf{x}_{k}(t+1)=\mathbf{T}_{k}(\mathbf{x}(t))$. The encoder $\mathcal{E}_{k}$ of $\mathcal{Q}_{k}$ accepts the input $\mathbf{T}_{k}(\mathbf{x}(t))$ and produces a quantization index $I_{k}(t)=\mathcal{E}_{k}\left(\mathbf{T}_{k}(\mathbf{x}(t))\right)$. Each node $k$ broadcasts the quantization index $I_{k}(t)$. In other words, the message passing involves only the quantization indices $\mathbf{I}(t)=\left(I_{k}(t), \cdots, I_{K}(t)\right)$ instead of the actual controls $\mathbf{T}(\mathbf{x}(t))=\left(\mathbf{T}_{1}(\mathbf{x}(t)), \cdots, \mathbf{T}_{K}(\mathbf{x}(t))\right)$. Upon receiving the quantization index $I_{k}(t)$, the decoder $\mathcal{D}_{k}$ of $\mathcal{Q}_{k}$ produces a quantized value $\mathbf{x}_{k}(t+1)=$ $\hat{\mathbf{T}}_{k}(\mathbf{x}(t))=\mathcal{D}_{k}\left(I_{k}(t)\right)=\mathbf{T}_{k}(\mathbf{x}(t))+\mathbf{e}_{k}(t)$. Therefore, the contracting iteration update dynamics of (1) with quantized message passing can be modified as:

$$
\mathbf{x}(t+1)=\mathbf{T}(\mathbf{x}(t))+\mathbf{e}(t)
$$

where $\mathbf{e}(t) \in \mathbb{R}^{n}$ is the quantization error vector at time $t$. The quantizer design affects the convergence property of the iterative update algorithm fundamentally via the quantization error random process $\mathbf{e}(t)$. Generally, the update of each block component is based on the latest overall vector, because $\mathbf{T}_{k}: \mathcal{X} \rightarrow \mathcal{X}_{k}$. Thus, the decoders of the system quantizer $\mathcal{D}=\left(\mathcal{D}_{1}, \cdots, \mathcal{D}_{K}\right)$ is needed at each node. On the other hand, the $k$-th node only requires the encoder $\mathcal{E}_{k}$ of the corresponding quantizer component $\mathcal{Q}_{k}$.

Consider the application example in Section I-C under quantized message passing. The system quantizer $\mathcal{Q}=$ $\left(\mathcal{Q}_{1}, \ldots, \mathcal{Q}_{K}\right)$ can be applied in the MIMO interference game with $K$ noncooperative transmitter-receiver pairs as illustrated in Fig. 1. Specifically, for the $k$-th link, the encoder $\mathcal{E}_{k}$ is placed at receiver and the decoder $\mathcal{D}_{k}$ is placed at the transmitter. The MIMO interference game under quantized message passing will be illustrated in the following example:

Example 1: (MIMO Interference under Quantized Message Passing) In the $t$-th iteration, the receiver of the $k$-th link locally measures PSD of the interference received from the transmitter of the other links, i.e. $\sum_{j \neq k} \mathbf{H}_{j k} \mathbf{P}_{j}(t) \mathbf{H}_{j k}^{H}$, and computes $\mathbf{W} \mathbf{F}_{k}\left(\mathbf{P}_{-k}(t)\right)$. 
The encoder $\mathcal{E}_{k}$ of $\mathcal{Q}_{k}$ at the $k$-th receiver encodes $\mathbf{W} \mathbf{F}_{k}\left(\mathbf{P}_{-k}(t)\right)$ and passes the quantization index $I_{k}(t)=\mathcal{E}_{k}\left(\mathbf{W F}_{k}\left(\mathbf{P}_{-k}(t)\right)\right)$ to the $k$-th transmitter. The decoder $\mathcal{D}_{k}$ of $\mathcal{Q}_{k}$ at $k$-th transmitter produces a quantized value $\mathbf{P}_{k}(t+1)=\mathcal{D}_{k}\left(I_{k}(t)\right)=\mathbf{W} \mathbf{F}_{k}\left(\mathbf{P}_{-k}(t)\right)+$ $\mathbf{e}_{k}(t)$. The contracting iterative update dynamics of 13 for the MIMO interference game under quantized message passing is given by:

$$
\mathbf{P}(t+1)=\mathbf{W F}(\mathbf{P}(t))+\mathbf{e}(t)
$$

\section{B. Convergence Property under Quantized Message Passing}

Under the quantized message passing, the convergence of the contracting iterations is summarized in the following lemma:

Lemma 1: (Convergence of Contracting Iterations under Quantized Message Passing) Suppose that $\mathbf{T}: \mathcal{X} \rightarrow$ $\mathcal{X}$ is a contraction mapping with modulus $\alpha \in[0,1)$ and fixed point $\mathrm{x}^{*} \in \mathcal{X}$, and that $\mathcal{X} \subseteq \mathbb{R}^{n}$ is closed. For any initial vector $\mathbf{x}(0) \in \mathcal{X}$, the sequence $\{\mathbf{x}(t)\}$ generated by (14) satisfies:

(a) $\left\|\mathbf{x}(t)-\mathbf{x}^{*}\right\| \leq \alpha^{t}\left\|\mathbf{x}(0)-\mathbf{x}^{*}\right\|+E(t) \forall t \geq 1$, where $E(t)=\alpha^{t-1} \sum_{l=0}^{t-1} \alpha^{-l}\|\mathbf{e}(l)\|$ is the accumulated error up to the time $t$ induced by the quantized message passing.

(b) For each $t$, if there exists a vector $\tilde{\mathbf{e}}_{t} \in \mathbb{R}^{n}$ such that $\|\mathbf{e}(t)\| \leq\left\|\tilde{\mathbf{e}}_{t}\right\|$, then $E(t) \leq \tilde{E}(t)$, where $\tilde{E}(t) \triangleq$ $\alpha^{t-1} \sum_{l=0}^{t-1} \alpha^{-l}\left\|\tilde{\mathbf{e}}_{l}\right\|$.

(c) If $\left\|\tilde{\mathbf{e}}_{1}\right\|=\cdots=\left\|\tilde{\mathbf{e}}_{t}\right\| \triangleq\|\overline{\mathbf{e}}\|$, then $E(t) \leq \bar{E}(t)$, where $\bar{E}(t) \triangleq \frac{1-\alpha^{t}}{1-\alpha}\|\overline{\mathbf{e}}\|$ with limiting error bound $\bar{E}(\infty) \triangleq \lim _{t \rightarrow \infty} \bar{E}(t)=\frac{\|\overline{\mathbf{e}}\|}{1-\alpha}$. Furthermore, define the stationary set as $\mathbb{S} \triangleq\left\{\mathcal{Q}(\mathbf{x}):\left\|\mathbf{x}-\mathbf{x}^{*}\right\| \leq \bar{E}(\infty)\right\}$. The sufficient condition for convergence is $\mathbf{x}=\mathcal{Q}(\mathbf{T}(\mathbf{x}))$ $\forall \mathbf{x} \in \mathbb{S}$ and the necessary condition for convergence is $\exists \mathrm{x} \in \mathbb{S}$, such that $\mathrm{x}=\mathcal{Q}(\mathbf{T}(\mathbf{x}))$.

Proof: Please refer to Appendix A for the proof.

Note that, in the above lemma, the norm $\|\cdot\|$ can be any general norm. In the following, we shall focus on characterizing the convergence behavior of the distributed Jacobi and Gauss-Seidel schemes under quantized message passing with the underlying contraction mapping $\mathbf{T}$ defined w.r.t. the weighted block-maximum norm $\|\cdot\|_{\text {block }}^{\mathrm{w}}[5]-[7]$. Under quantized message passing, the algorithm dynamics of the two commonly used parallel and distributed schemes can be described as follows:

- Jacobi Scheme under Quantized Message Passing:

$$
\mathbf{x}_{k}(t+1)=\mathbf{T}_{k}(\mathbf{x}(t))+\mathbf{e}_{k}(t), 1 \leq k \leq K
$$

- Gauss-Seidel Scheme under Quantized Message Passing:

$$
\mathbf{x}_{k}(t+1)=\hat{\mathbf{S}}_{k}(\mathbf{x}(t))+\mathbf{e}_{k}(t), 1 \leq k \leq K
$$

where

$$
\begin{aligned}
& \hat{\mathbf{S}}_{k}(\mathbf{x}) \\
& = \begin{cases}\mathbf{T}_{k}(\mathbf{x}), & k=1 \\
\mathbf{T}_{k}\left(\hat{\mathbf{S}}_{1}(\mathbf{x})+\mathbf{e}_{1}, \cdots,\right. & \\
\left.\hat{\mathbf{S}}_{k-1}(\mathbf{x})+\mathbf{e}_{k-1}, \mathbf{x}_{k}, \cdots, \mathbf{x}_{K}\right), & 2 \leq k \leq K\end{cases}
\end{aligned}
$$

Applying the results of Lemma 1 the convergence property of the distributed Jacobi and Gauss-Seidel schemes in (16) and (17) can be summarized in the following lemma.

Lemma 2: (Convergence of Jacobi Scheme and Gauss-Seidel Scheme under Quantized Message Passing) Suppose that $\mathbf{T}: \mathcal{X} \rightarrow \mathcal{X}$ is a block-contraction mapping w.r.t. the weighted block-maximum norm $\|\cdot\|_{\text {block }}^{\mathbf{w}}$ with modulus $\alpha \in[0,1)$ and fixed point $\mathrm{x}^{*} \in \mathcal{X}$, and that $\mathcal{X} \subseteq \mathbb{R}^{n}$ is closed. For every initial vector $\mathrm{x}(0) \in \mathcal{X}$, the sequence $\{\mathbf{x}(t)\}$ generated by both the Jacobi scheme and the Gauss-Seidel scheme under quantized message passing in (16) and (17) satisfies 5

(a) $\left\|\mathbf{x}(t)-\mathbf{x}^{*}\right\|_{\text {block }}^{\mathbf{w}} \leq \alpha^{t}\left\|\mathbf{x}(0)-\mathbf{x}^{*}\right\|_{\text {plock }}^{\mathbf{w}}+E_{\text {block }}^{\mathbf{w}}(t)$ $\forall t \geq 1$, where $E_{\text {block }}^{\mathbf{w}}(t)=\alpha^{t-1} \sum_{l=0}^{t-1} \alpha^{-l}\|\mathbf{e}(l)\|_{\text {block }}^{\mathbf{w}}$ for Jacobi scheme and $E_{\text {block }}^{\mathbf{w}}(t)=$ $\frac{1-\alpha^{K}}{1-\alpha} \alpha^{t-1} \sum_{l=0}^{t-1} \alpha^{-l}\|\mathbf{e}(l)\|_{\text {block }}^{\mathbf{w}} \quad$ for $\quad$ Gauss-Seidel scheme.

(b) If condition in (b) of Lemma 1 holds w.r.t. $\|\cdot\|_{\text {block }}^{\mathbf{w}}$, then $E_{\text {block }}^{\mathbf{w}}(t) \leq \tilde{E}_{\text {block }}^{\mathbf{w}}(t)$, where $\tilde{E}_{\text {block }}^{\mathbf{w}}(t) \triangleq \alpha^{t-1} \sum_{l=0}^{t-1} \alpha^{-l}\left\|\tilde{\mathbf{e}}_{l}\right\|_{\text {block }}^{\mathbf{w}}$ for Jacobi scheme and $\tilde{E}_{\text {block }}^{\mathbf{w}}(t) \triangleq \frac{1-\alpha^{K}}{1-\alpha} \alpha^{t-1} \sum_{l=0}^{t-1} \alpha^{-l}\left\|\tilde{\mathbf{e}}_{l}\right\|_{\text {block }}^{\mathbf{w}}$ for Gauss-Seidel scheme.

(c) If condition in (c) of Lemma 11 holds w.r.t. $\|\cdot\|_{\text {block }}^{\mathbf{w}}$, then $E_{\text {block }}^{\mathbf{w}}(t) \leq \bar{E}_{\text {block }}^{\mathbf{w}}(t)$, where $\bar{E}_{\text {block }}^{\mathbf{w}}(t) \triangleq$ $\frac{1-\alpha^{t}}{1-\alpha}\|\overline{\mathbf{e}}\|_{\text {block }}^{\mathbf{w}}$ for Jacobi scheme with $\bar{E}_{\text {block }}^{\mathbf{w}}(\infty)=$ $\frac{1}{1-\alpha}\|\overline{\mathbf{e}}\|_{\text {block }}^{\mathbf{w}}$ and $\bar{E}_{\text {block }}^{\mathbf{w}}(t) \triangleq \frac{1-\alpha^{K}}{1-\alpha} \frac{1-\alpha^{t}}{1-\alpha}\|\overline{\mathbf{e}}\|_{\text {block }}^{\mathbf{w}}$ for Gauss-Seide 6 scheme with $\bar{E}_{\text {block }}^{\mathbf{w}}(\infty)=\frac{1-\alpha^{K}}{(1-\alpha)^{2}}\|\overline{\mathbf{e}}\|_{\text {block }}^{\mathbf{w}}$. Furthermore, define the stationary set as $\mathbb{S}_{\text {block }}^{\mathrm{w}} \triangleq$ $\left\{\mathcal{Q}(\mathrm{x}):\left\|\mathrm{x}-\mathrm{x}^{*}\right\|_{\text {block }}^{\mathbf{w}} \leq \bar{E}_{\text {block }}^{\mathbf{w}}(\infty)\right\}$. The sufficient condition and necessary condition are the same as those in Lemma 1

Proof: Please refer to Appendix B for the proof.

\footnotetext{
${ }^{5}$ Our analysis can be extended to totally asynchronous scheme in which the results of Lemma 2 becomes: (a) $E_{\text {block }}^{\mathbf{w}}(t)=\frac{1}{1-\alpha} \alpha^{t-1} \sum_{l=0}^{t-1} \alpha^{-l}\|\mathbf{e}(l)\|_{\text {block }}^{\mathbf{w}}$ (b) $\tilde{E}_{\text {block }}^{\mathbf{w}}(t) \triangleq \frac{1}{1-\alpha} \alpha^{t-1} \sum_{l=0}^{t-1} \alpha^{-l}\left\|\tilde{\mathbf{e}}_{l}\right\|_{\text {block }}^{\mathbf{w}}$. (c) If $\|\overline{\mathbf{e}}\|_{\text {block }}^{\mathbf{w}}<$ $(1-\alpha)\left\|\mathbf{x}(0)-\mathbf{x}^{*}\right\|_{\text {block }}^{\mathbf{w}}$, we have $\bar{E}_{\text {block }}^{\mathbf{w}}(t)=\frac{1-\alpha^{t}}{(1-\alpha)^{2}}\|\overline{\mathbf{e}}\|_{\text {block }}^{\mathbf{w}}$ and $\bar{E}_{\text {block }}^{\mathbf{w}}(\infty)=\frac{1}{(1-\alpha)^{2}}\|\overline{\mathbf{e}}\|_{\text {block. }}^{\mathbf{w}}$. By the Asynchronous Convergence Theorem (Proposition 2.1 in Chapter 6 of [7]), we can prove (c) (similar to the proof of Theorem 12 in [6]). The proof is omitted here due to page limit. Since the error bound result of totally asynchronous scheme is similar to Jacobi Scheme and Gauss-Seidel Scheme, our quantizer design later can be applied to the asynchronous case.

${ }^{6}$ Compared with Jacobi scheme, Gauss-Seidel scheme and totally asynchronous scheme have extra error terms $\frac{1-\alpha^{K}}{1-\alpha}$ and $\frac{1}{1-\alpha}$, respectively, and $\frac{1}{1-\alpha}>\frac{1-\alpha^{K}}{1-\alpha}>1$.
} 
Remark 2: As a result of Lemma1 1 and Lemma 2, the effect of quantized message passing affects the convergence property of the contracting iterative algorithm in a fundamental way. From Lemma 2 the Jacobi and GaussSeidel distributed iterative algorithms may not be able to converge precisely to the fixed point under quantized message passing due to the term $E_{\mathrm{block}}^{\mathrm{w}}(t)$.

\section{Time InVariant Convergence-OptimaL QUANTIZER DESIGN}

In this section, we shall define a Time Invariant Quantizer (TIQ) and then formulate the Time Invariant Convergence-Optimal Quantizer (TICOQ) design problem. We shall consider the TICOQ design for the scalar quantizer (SQ) and the vector quantizer (VQ) cases separately. Specifically, the component quantizer $\mathcal{Q}_{k}$ of the $k$-th node can be a group of scalar quantizers $\mathcal{Q}_{k}^{s}=\left(\mathcal{Q}_{m}^{s}\right)_{m \in \mathcal{M}_{k}}$ or a vector quantizer $\mathcal{Q}_{k}^{v}$. In the SQ case, each element $T_{m}(\cdot)\left(m \in \mathcal{M}_{k}\right)$ of the vector $\mathbf{T}_{k}(\cdot)$ is quantized by a coordinate scalar quantizer $Q_{m}^{s}$ separately. However, in the VQ case, the input to a vector quantizer $Q_{k}^{v}$ is the vector $\mathbf{T}_{k}(\cdot)$.

Definition 2 (Time Invariant Quantizer (TIQ)):

A Time Invariant Quantizer (TIQ) is a quantizer $\mathcal{Q}=(\mathcal{E}, \mathcal{D})$ such that $\mathcal{E}$ and $\mathcal{D}$ are time invariant mappings.

The system scalar TIQ can be denoted as $\mathcal{Q}^{s}=$ $\left(\mathcal{Q}_{1}^{s}, \cdots, \mathcal{Q}_{m}^{s}, \cdots, \mathcal{Q}_{n}^{s}\right)$. Let $\mathbf{L}^{s}=\left(L_{1}^{s}, \cdots, L_{n}^{s}\right)$ be the quantization rate vector for the system scalar TIQ $\mathcal{Q}^{s}$, where $L_{m}^{s} \in \mathbb{Z}^{+}$is the quantization rate (number of bits) of the coordinate scalar quantizer $\mathcal{Q}_{m}^{s}(1 \leq m \leq n)$. The sum quantization rate of the system scalar TIQ $\mathcal{Q}^{s}$ is given by $\sum_{m=1}^{n} L_{m}^{s}$. Similarly, the system vector TIQ can be denoted as $\mathcal{Q}^{v}=\left(\mathcal{Q}_{1}^{v}, \cdots, \mathcal{Q}_{k}^{v}, \cdots, \mathcal{Q}_{K}^{v}\right)$. Let $\mathbf{L}^{v}=\left(L_{1}^{v}, \cdots, L_{K}^{v}\right)$ be the quantization rate vector for the system vector TIQ $\mathcal{Q}^{v}$, where $L_{k}^{v} \in \mathbb{Z}^{+}$is defined as the quantization rate (number of bits) of the coordinate vector quantizer $\mathcal{Q}_{k}^{v}(1 \leq k \leq K)$. The sum quantization rate of the system vector TIQ $\mathcal{Q}^{v}$ is given by $\sum_{k=1}^{K} L_{k}^{v}$.

Using Lemma2 (c), the limiting error bound of the algorithm trajectory is given by $\bar{E}_{\text {block }}^{\mathbf{w}}(\infty)=\frac{1}{1-\alpha}\|\overline{\mathbf{e}}\|_{\text {block }}^{\mathbf{w}}$ (Jacobi scheme) or $\bar{E}_{\text {block }}^{\mathbf{w}}(\infty)=\frac{1-\alpha^{K}}{(1-\alpha)^{2}}\|\overline{\mathbf{e}}\|_{\text {block }}^{\mathbf{w}}$ (GaussSeidel scheme). Therefore, the TICOQ design, which minimizes $\bar{E}_{\text {block }}^{\mathbf{w}}(\infty)$ under the sum quantization rate constraint, is equivalent to the following:

Problem 1 (TICOQ Design Problem):

$$
\begin{aligned}
\min _{\mathcal{Q}^{s} \text { or } \mathcal{Q}^{v}} & \|\overline{\mathbf{e}}\|_{\text {block }}^{\mathbf{w}} \\
\text { s.t. } & \sum_{m=1}^{n} L_{m}^{s}=L, L_{m}^{s} \in \mathbb{Z}^{+}(1 \leq m \leq n) \mathrm{SQ} \\
& \text { or } \sum_{k=1}^{K} L_{k}^{v}=L, L_{k}^{v} \in \mathbb{Z}^{+}(1 \leq k \leq K) \mathrm{VQ}
\end{aligned}
$$

where $\|\overline{\mathbf{e}}\|_{\text {block }}^{\mathbf{w}}=\max _{\mathbf{x} \in \mathcal{X}}\left\|\mathbf{x}-\mathcal{Q}^{s}(\mathbf{x})\right\|_{\text {block }}^{\mathbf{w}}$ (SQ case) or $\|\overline{\mathbf{e}}\|_{\text {block }}^{\mathbf{w}}=\max _{\mathbf{x} \in \mathcal{X}}\left\|\mathbf{x}-\mathcal{Q}^{v}(\mathbf{x})\right\|_{\text {block }}^{\mathbf{w}}$ (VQ case).

Remark 3 (Interpretation of Problem [1: Note that the optimization variable in Problem 1 is the system TIQ $\mathcal{Q}^{s}$ or $\mathcal{Q}^{v}$. The objective function $\|\overline{\mathbf{e}}\|_{\text {block }}^{\mathbf{w}}=\max _{\mathbf{x} \in \mathcal{X}}\left\|\mathbf{x}-\mathcal{Q}^{s}(\mathbf{x})\right\|_{\text {block }}^{\mathbf{w}}$ or $\|\overline{\mathbf{e}}\|_{\text {block }}^{\mathbf{w}}=\max _{\mathbf{x} \in \mathcal{X}}\left\|\mathbf{x}-\mathcal{Q}^{v}(\mathbf{x})\right\|_{\text {block }}^{\mathbf{w}}$ obviously depends on the choice of the system TIQ $\mathcal{Q}^{s}$ or $\mathcal{Q}^{v}$. Furthermore, the constraint (20) or (21) is the constraint on the quantization rate $\mathbf{L}^{s}=\left(L_{1}^{s}, \cdots, L_{n}^{s}\right)$ or $\mathbf{L}^{v}=\left(L_{1}^{v}, \cdots, L_{K}^{v}\right)$, which is also an effective constraint on the optimization domains of $\mathcal{Q}^{s}$ or $\mathcal{Q}^{v}$, respectively. It is because $L_{m}^{s}$ or $L_{k}^{v}$ is a parameter (corresponding to the cardinality of the index set, i.e. $\left|\mathcal{I}_{m}^{s}\right|=2^{L_{m}^{s}}$ or $\left|\mathcal{I}_{k}^{v}\right|=2^{L_{k}^{v}}$ ) of the encoder and decoder of $\mathcal{Q}_{m}^{s}$ or $\mathcal{Q}_{k}^{v}$. The Lagrangian function of Problem 1 is given by: $\mathcal{L}^{s}\left(\mathcal{Q}^{s}, \mu^{s}\right)=\|\overline{\mathbf{e}}\|_{\text {block }}^{\mathbf{w}}+\mu^{s}\left(\sum_{m=1}^{n} L_{m}^{s}-L\right)$ (SQ case) or $\mathcal{L}^{v}\left(\mathcal{Q}^{v}, \mu^{v}\right)=\|\overline{\mathbf{e}}\|_{\text {block }}^{\mathbf{w}}+\mu^{v}\left(\sum_{k=1}^{K} L_{k}^{v}-L\right)$ (VQ case), where $\mu^{s}$ or $\mu^{v}$ is the Lagrange multiplier (LM) corresponding to the constraint (20) or (21). Hence, the optimization problem 1 can also be interpreted as optimizing the tradeoff between the convergence performance $\|\overline{\mathbf{e}}\|_{\text {block }}^{\mathbf{w}}$ and the communication overhead $\sum_{m=1}^{n} L_{m}^{s}$ or $\sum_{k=1}^{K} L_{k}^{v}$. The LM $\mu^{s}$ or $\mu^{v}$ can be regarded as the per-iteration cost sensitivity.

Remark 4 (Robust Consideration in Problem 17):

The optimization objective $\|\overline{\mathbf{e}}\|_{\text {block }}^{\mathbf{w}}$ in (19) actually corresponds to a worst case error in the algorthm trajectory. In other words, the TICOQ design is trying to find the optimal TIQ which minimizes the worst case error. In fact, the algorithm trajectory $\mathbf{x}(t)$ is a random process induced by the uncertainty in the initial point $\mathbf{x}(0)$. In general, we do not have knowledge on the distribution of $\mathbf{x}(t)$ due to the uncertainty on $\mathbf{x}(0)$. Hence, the solution to Problem 1 (optimizing the worst case error) offers some robustness w.r.t. the choice of $\mathbf{x}(0)$.

In the following, we shall discuss the scalar and vector TICOQ design based on Problem 1 separately.

\section{A. Time Invariant Convergence-Optimal Scalar Quan- tizer}

We first have a lemma on the structure of the optimizing quantizer in the scalar TICOQ design in Problem 1

Lemma 3 (Structure of the Scalar TICOQ): If each component norm $\|\cdot\|_{k}$ on $\mathbb{R}^{n_{k}}(1 \leq k \leq K)$ of the weighted block-maximum norm $\|\cdot\|_{\text {block }}^{\mathbf{w}}$ defined by (7) is monotone (or absolute) norm 7 , then the optimal coordinate scalar quantizer $\mathcal{Q}_{m}^{s *}(1 \leq m \leq n)$ w.r.t. the worst case error $\|\overline{\mathbf{e}}\|_{\text {block }}^{\mathbf{w}}=\max _{\mathbf{x} \in \mathcal{X}}\left\|\mathbf{x}-\mathcal{Q}^{s}(\mathbf{x})\right\|_{\text {block }}^{\mathbf{w}}$ is a uniform quantizer.

\footnotetext{
${ }^{7} \mathrm{~A}$ vector norm is monotone if and only if it is absolute [23].
} 
Proof: Please refer to Appendix C for the proof.

While the optimization variable in Problem 1 (SQ case) is $\mathcal{Q}^{s}=\left(Q_{1}^{s}, \cdots, Q_{n}^{s}\right)$, using Lemma 3, we can restrict the optimization domain of each coordinate scalar quantizer $\mathcal{Q}_{m}^{s}(1 \leq m \leq n)$ to uniform quantizer without loss of optimality. Thus, the worst case error of the $m$-th coordinate is given by $\left|\bar{e}_{m}\right| \triangleq$ $\max _{x_{m} \in \mathcal{X}_{m}}\left|x_{m}-\mathcal{Q}_{m}^{s}\left(x_{m}\right)\right|=\frac{\left|\mathcal{X}_{m}\right|}{2 \times 2^{L s}}(1 \leq m \leq n)$, where $\left|\mathcal{X}_{m}\right|$ is the length of the interval $\mathcal{X}_{m}\left(x_{m} \in \mathcal{X}_{m}\right)$, and the remaining optimization variable is reduced from $\mathcal{Q}^{s}=\left(Q_{1}^{s}, \cdots, Q_{n}^{s}\right)$ to $\mathbf{L}^{s}=\left(L_{1}^{s}, \cdots, L_{n}^{s}\right)$. Scalar TICOQ design in Problem 1 w.r.t. $\mathbf{L}^{s}=\left(L_{1}^{s}, \cdots, L_{n}^{s}\right)$ is a Nonlinear Integer Programming (NLIP) problem, which is in general difficult to solve. Verifying the optimality of a solution requires enumerating all the feasible solutions in most cases. In the following, we shall derive the optimal solution to the scalar TICOQ design in Problem 1 w.r.t. the weighted block-maximum norm $\|\cdot\|_{\text {block }}^{\mathbf{w}}$ defined by (7), in which each component norm $\|\cdot\|_{k}$ is the weighted maximum norm and the $L_{p}$ norm separately.

Theorem 3 (Solution for Weighted Maximum Norm): Given a weighted block-maximum norm $\|\cdot\|_{\text {block }}^{\mathbf{w}}$ defined by (7) (parameterized by $\mathbf{w}=\left(w_{1}, \cdots, w_{K}\right)$ ) with $\|\cdot\|_{k}$ being the weighted maximum norm $\|\cdot\|_{\infty}^{\mathbf{a}_{k}}$ defined by (2) (parameterized by $\mathbf{a}_{k}=\left(a_{m}\right)_{m \in \mathcal{M}_{k}}$ ), let $\bar{L}_{m}^{s *}=\left(\log _{2} \frac{C_{m}}{\tau}\right)^{+}$, where $C_{m} \triangleq \frac{\left|\mathcal{X}_{m}\right|}{2 a_{m}\left(\sum_{k=1}^{K} w_{k} \mathbf{I}\left[m \in \mathcal{M}_{k}\right]\right)}$, $\mathbf{I}[\cdot]$ is indicator function and $\tau>0$ is a constant related to the LM of the constraint (20) chosen to satisfy the constraint $\sum_{m=1}^{n}\left(\log _{2} \frac{C_{m}}{\tau}\right)^{+}=L$. The optimal integer solution of Problem 1 for the SQ case is given by 8 :

$$
L_{[m]}^{s *}= \begin{cases}\left\lceil\bar{L}_{[m]}^{s *}\right], & \text { if } m \leq \sum_{m=1}^{n}\left(\bar{L}_{m}^{s *}-\left\lfloor\bar{L}_{m}^{s *}\right\rfloor\right) \\ \left\lfloor\bar{L}_{[m]}^{s *}\right\rfloor, & \text { otherwise }\end{cases}
$$

The optimal value of Problem 1 under continuous relaxation is $\tau$.

Proof: Please refer to Appendix D for the Proof.

Theorem 4 (Solution for $L_{p}$ Norm): Given weighted block-maximum norm $\|\cdot\|_{\text {block }}^{\mathbf{w}}$ defined by (7) (parameterized by $\left.\mathbf{w}=\left(w_{1}, \cdots, w_{K}\right)\right)$ with $\|\cdot\|_{k}$ being the $L_{p}$ norm $\|\cdot\|_{p}$ defined by (3) (parameterized by $p$ ), the optimal solution of Problem 1 for the SQ case with continuous relaxation $\left(L_{m}^{s} \in \mathbb{R}^{+}\right)$is $\bar{L}_{m}^{s *}=\frac{1}{p} \log _{2}\left(\frac{C_{m}}{\sum_{k=1}^{K} \tau_{k} \mathbf{I}\left[m \in \mathcal{M}_{k}\right]} \vee 1\right)$, where $C_{m} \triangleq \frac{\left|\mathcal{X}_{m}\right|^{p}}{2^{p}\left(\sum_{k=1}^{K} w_{k}^{p} \mathbf{I}\left[m \in M_{k}\right]\right)}, \quad\left\{\tau_{1}, \cdots, \tau_{K}\right\}$ and $\tau$ are constants related to the LM of the constraint (21) chosen to satisfy the constraint $\sum_{k=1}^{K} \sum_{m \in \mathcal{M}_{k}} \frac{1}{p} \log _{2}\left(\frac{C_{m}}{\tau_{k}} \vee 1\right)=L$ and complementary slackness conditions $\frac{1}{\tau_{k}}\left(\sum_{m \in \mathcal{M}_{k}}\left(C_{m} \wedge \tau_{k}\right)-\tau\right)=0$

\footnotetext{
${ }^{8} \mathrm{We}$ arrange the real sequence $z_{1}, \cdots, z_{n}$ in decreasing order and denote them as $z_{[1]} \geq \cdots \geq z_{[m]} \geq \cdots \geq z_{[n]}$, where $z_{[m]}$ represents the $m$-th largest term of $\left\{z_{m}\right\}$.
}

$(\forall k \in\{1, \cdots, K\}$. The optimal value of Problem 1 with continuous relaxation is $\tau^{\frac{1}{p}}$.

Proof: Please refer to Appendix D for the Proof.

Remark 5 (Determination of $\left\{\tau_{1}, \cdots, \tau_{K}\right\}$ and $\tau$ ):

Solving for $\left\{\tau_{1}, \cdots, \tau_{K}\right\}$ and $\tau$ involves solving a system of $K+1$ equations with $K+1$ unknowns. We have $2^{K}-1$ valid cases for the above system of equations according to $\tau_{k}>\max _{m \in \mathcal{M}_{k}} C_{m}$ or $\tau_{k} \leq \max _{m \in \mathcal{M}_{k}} C_{m}(\forall k)$. Firstly, if $\tau_{k}>\max _{m \in \mathcal{M}_{k}} C_{m}(\forall k)$, then $\bar{L}_{m}^{s}=0$ $(\forall m)$, which is not a valid case. Therefore, without loss of generality, assume $\tau_{k} \leq \max _{m \in \mathcal{M}_{k}} C_{m}$ $\forall k \in\{1, \cdots, N\}$ and $\tau_{k}>\max _{m \in \mathcal{M}_{k}} C_{m}$ $\forall k \in\{N+1, \cdots, K\}$. The system of $K+1$ equations and unknowns reduce to $N+1$ equations, which are given by: $\sum_{k=1}^{N} \sum_{m \in \mathcal{M}_{k}} \frac{1}{p} \log _{2}\left(\frac{C_{m}}{\tau_{k}} \vee 1\right)=L$ and $\sum_{m \in \mathcal{M}_{k}}\left(C_{m} \wedge \tau_{k}\right)=\tau(\forall k \stackrel{p}{\in}\{1, \cdots, N\})$, and $N+1$ unknown 10: $\left\{\tau_{1}, \cdots, \tau_{N}\right\}$ and $\tau$.

\section{B. Time Invariant Convergence-Optimal Vector Quan- tizer}

We first have a lemma on the structure of the optimizing quantizer in the vector TICOQ design in Problem 1

Lemma 4 (Structure of the Vector TICOQ): If the component norm $\|\cdot\|_{k}(1 \leq k \leq K)$ on $\mathbb{R}^{n_{k}}$ of the weighted block-maximum norm $\|\cdot\|_{\text {block }}^{\mathrm{w}}$ defined by (7) is monotone (or absolute) norm, each vector TICOQ $\mathcal{Q}_{k}^{v *}$ is a $n_{k}$-dimensional lattice quantizer, the structure of which is uniquely determined by the norm $\|\cdot\|_{k}$ on $\mathbb{R}^{n_{k}}$. In particular, if $\|\cdot\|_{k}(1 \leq k \leq K)$ is $L_{2}$ norm, each vector TICOQ $\mathcal{Q}_{k}^{v *}$ is the thinnest lattice for the covering problem in Euclidean space; If $\|\cdot\|_{k}$ $(1 \leq k \leq K)$ is weighted maximum norm, each vector TICOQ $\mathcal{Q}_{k}^{v *}$ reduces to $n_{k}$ coordinate scalar TICOQ $\mathcal{Q}_{m}^{s *}\left(m \in \mathcal{M}_{k}\right)$ with scalar quantization 11 of each coordinate $x_{m}\left(m \in \mathcal{M}_{k}\right)$ of $\mathbf{x}_{k}$.

Proof: Please refer to Appendix E for the proof.

A lattice is a regular arrangement of points in $n$ dimensional space that includes the origin. "Regular" means that each point "sees" the same geometrical environment as any other point [20]. The lattice covering

$$
{ }^{9} x \vee a \triangleq \max \{x, a\} \text { and } x \wedge a \triangleq \min \{x, a\} .
$$

${ }^{10}$ For example, consider $K=2, n=4, \mathcal{M}_{1}=\{1,2\}$ and $\mathcal{M}_{2}=\{3,4\}$. We have $2^{2}-1=3$ valid cases. Case 1 ( $\tau_{1} \leq \max _{m \in \mathcal{M}_{1}} C_{m}$ and $\left.\tau_{2} \leq \max _{m \in \mathcal{M}_{2}} C_{m}\right)$ We have 3 equations: $\sum_{k=1}^{2} \sum_{m \in \mathcal{M}_{k}} \frac{1}{p} \log _{2}\left(\frac{C_{m}}{\tau_{k}} \vee 1\right)=L, \sum_{m \in \mathcal{M}_{k}}\left(C_{m} \wedge\right.$ $\left.\tau_{k}\right)=\tau(\forall k \in\{1,2\})$, and 3 unknowns: $\left\{\tau_{1}, \tau_{2}\right\}, \tau$. Case 2( $\tau_{1} \leq \max _{m \in \mathcal{M}_{1}} C_{m}$ and $\left.\tau_{2}>\max _{m \in \mathcal{M}_{2}} C_{m}\right)$ We have 2 equations: $\sum_{m \in \mathcal{M}_{1}} \frac{1}{p} \log _{2}\left(\frac{C_{m}}{\tau_{1}} \vee 1\right)+0+0=L, \sum_{m \in \mathcal{M}_{1}}\left(C_{m} \wedge\right.$ $\left.\tau_{1}\right)=\tau$, and 2 unknowns: $\tau_{1}, \tau$. Case $3\left(\tau_{1}>\max _{m \in \mathcal{M}_{1}} C_{m}\right.$ and $\tau_{2} \leq \max _{m \in \mathcal{M}_{2}} C_{m}$ ) We have 2 equations: $0+0+$ $\sum_{m \in \mathcal{M}_{2}} \frac{1}{p} \log _{2}\left(\frac{C_{m}}{\tau_{2}} \vee 1\right)=L, \sum_{m \in \mathcal{M}_{2}}\left(C_{m} \wedge \tau_{2}\right)=\tau$, and 2 unknowns: $\tau_{2}, \tau$.

${ }^{11}$ In other words, the vector TICOQ design reduces to scalar TICOQ design discussed in Theorem 3 
problem asks for the most economical way to arrange the lattice points so that the $n$-dimensional space can be covered with overlapping spheres whose centers are the lattice points, i.e. tries to find the thinnest (i.e. minimum density 12 ) lattice covering [24]. The thinnest lattice coverings known in all dimension $n(n \leq 23)$ are the dual lattice $A_{n}^{*}$ (when $1 \leq n \leq 5, A_{n}^{*}$ is known to be optimal) [24]. The worst case error of the dual lattice $A_{n_{k}}^{*}$ for the $k$-th node when $\|\cdot\|_{k}$ is $L_{p}$ norm $(p>2)$ is less than that measured in $L_{2}$ norm (Appendix E). Therefore, if $\|\cdot\|_{k}$ is $L_{p}$ norm $(p \geq 2)$, we can solve the TICOQ design (VQ case) in Problem 1 using dual lattice structure $A_{n_{k}}^{*}$. Thus, the worst case error is given by $\left\|\overline{\mathbf{e}}_{k}\right\|_{k}=\left(\frac{\prod_{m \in \mathcal{M}_{k}}\left|\mathcal{X}_{m}\right|}{\sqrt{\frac{1}{n_{k}+1}}}\right)^{\frac{1}{n_{k}}} \sqrt{\frac{n_{k}\left(n_{k}+2\right)}{12\left(n_{k}+1\right)}} 2^{-\frac{L_{k}^{v}}{n_{k}}}$ (Appendix E), where $\left|\mathcal{X}_{m}\right|$ is the length of the interval $\mathcal{X}_{m}\left(x_{m} \in \mathcal{X}_{m}\right)(1 \leq m \leq n)$, and the remaining optimization variable is reduced from $\mathcal{Q}^{v}=\left(Q_{1}^{v}, \cdots, Q_{K}^{v}\right)$ to $\mathbf{L}^{v}=\left(L_{1}^{v}, \cdots, L_{K}^{v}\right)$. Similarly, vector TICOQ design in Problem 1 w.r.t. $\mathbf{L}^{v}=\left(L_{1}^{v}, \cdots, L_{K}^{v}\right)$ is also a Nonlinear Integer Programming (NLIP) problem, which is in general difficult to solve. In the following, we shall derive the optimal solution to the vector TICOQ design in Problem 1 based on dual lattice $A_{n_{k}}^{*}$.

Theorem 5 (Solution for Dual Lattice quantizer): Given a weighted block-maximum norm $\|\cdot\|_{\text {block }}^{\mathbf{w}}$ defined by (7) (parameterized by $\mathbf{w}=\left(w_{1}, \cdots, w_{K}\right)$ ) and the dual lattice $\left\{A_{n_{k}}^{*}: 1 \leq k \leq K\right\}$ quantizer, let $\bar{L}_{k}^{v *}=n_{k}\left(\log _{2} \frac{D_{k}}{\tau}\right)^{+}$, where $D_{k} \triangleq \frac{1}{w_{k}}\left(\frac{\prod_{m \in \mathcal{M}_{k}}\left|\mathcal{X}_{m}\right|}{\sqrt{\frac{1}{n_{k}+1}}}\right)^{\frac{1}{n_{k}}} \sqrt{\frac{n_{k}\left(n_{k}+2\right)}{12\left(n_{k}+1\right)}}$, and $\tau>0$ is a constant related to the LM of the constraint (21) chosen to satisfy the constraint $\sum_{k=1}^{K} n_{k}\left(\log _{2} \frac{D_{k}}{\tau}\right)^{+}=L$. The optimal integer solution of Problem 1 for the VQ case w.r.t. dual lattice $\left\{A_{n_{k}}^{*}: 1 \leq k \leq K\right\}$ quantizer when $n_{1}=\cdots=n_{K}$ is given by:

$$
L_{[k]}^{v *}= \begin{cases}\left\lceil\bar{L}_{[k]}^{v *}\right], & \text { if } k \leq \sum_{k=1}^{K}\left(\bar{L}_{k}^{v *}-\left\lfloor\bar{L}_{k}^{v *}\right\rfloor\right) \\ \left\lfloor\bar{L}_{[k]}^{v *}\right], & \text { otherwise }\end{cases}
$$

The optimal value of Problem 1 under continuous relaxation is $\tau$.

Proof: Please refer to Appendix E for the Proof.

\section{Tradeoff between Convergence Error and Message Passing Overhead}

In this subsection, we shall quantify the tradeoff between the convergence error of the algorithm trajectory and the message passing overhead using TICOQ. Specifically, the steady-state convergence error in the algorithm trajectory is related to $\bar{E}_{\text {block }}^{\mathbf{w}}(\infty)$ and the message passing overhead is related to the sum quantization rate

\footnotetext{
${ }^{12}$ The density of a covering is the defined as the number of spheres that contain a point of the space [24].
}

(number of bits) $L$ of the system quantizer. The following lemma summarizes the tradeoff result.

Lemma 5: (Performance Tradeoff of the Scalar and Vector TICOQ) For $L \geq L^{\prime}\left(L \in \mathbb{Z}^{+}\right)$, where

$L^{\prime}=\left\{\begin{array}{l}\sum_{m=1}^{n} \log _{2} C_{m}-n \log _{2}\left(\min _{m} C_{m}\right), \text { WM norm } \\ \sum_{m=1}^{n} \log _{2} \tilde{C}_{m}-n \log _{2}\left(\min _{m} \tilde{C}_{m}\right), L_{p} \text { norm } \\ \sum_{k=1}^{K} \log _{2} D_{k}-K \log _{2}\left(\min _{k} D_{k}\right), \text { dual lattice }\end{array}\right.$

$\tilde{C}_{m}=\left(\sum_{k=1}^{K} n_{k} \mathbf{1}\left[m \in \mathcal{M}_{k}\right]\right) C_{m}$, the limiting error bound of the scalar and vector TICOQ considered in this section is given by $\bar{E}_{\text {block }}^{\mathrm{w}}(\infty)=\frac{1}{1-\alpha} \mathcal{O}\left(2^{-\frac{L}{n}}\right)$.

Proof: Please refer to Appendix F for the proof.

Remark 6: As $L \rightarrow \infty, \bar{E}_{\text {block }}^{\mathbf{w}}(\infty) \rightarrow 0$, which reduces to the conventional convergence results with perfect message passing. On the other hand, $\bar{E}_{\text {block }}^{\mathbf{w}}(\infty)>0$ for finite $L$ and hence, we cannot guarantee the convergence behavior of the contracting iterations with TICOQ to the fixed point $\mathrm{x}^{*}$ of the contraction mapping $\mathbf{T}$ anymore. Nevertheless, the convergence error decreases exponentially w.r.t. the message passing overhead $L$.

\section{TIME VARYing CONVERGENCE-OPTIMAL QUANTIZER DESIGN}

Similar to Section D we shall define a Time Varying Quantizer (TVQ) and then formulate the Time Varying Convergence-Optimal Quantizer (TVCOQ) design problem. We shall consider the TVCOQ design for both the SQ and VQ cases separately.

Definition 3 (Time Varying Quantizer (TVQ)):

A Time Varying Quantizer (TVQ) is a quantizer $\mathcal{Q}(t)=(\mathcal{E}(t), \mathcal{D}(t))$ such that $\mathcal{E}(t)$ and $\mathcal{D}(t)$ changes with time. In other words, the quantizer $\mathcal{Q}(t)=(\mathcal{E}(t), \mathcal{D}(t))$ at the $t$-th iteration is function of time $t$.

The system scalar TVQ at the $t$-th iteration can be denoted as $\mathcal{Q}^{s}(t)=\left(\mathcal{Q}_{1}^{s}(t), \cdots, \mathcal{Q}_{m}^{s}(t), \cdots, \mathcal{Q}_{n}^{s}(t)\right)$ with quantization rate vector (at the $t$-th iteration) $\mathbf{L}^{s}(t)=\left(L_{1}^{s}(t), \cdots, L_{n}^{s}(t)\right)$. Similarly, the system vector TVQ at the $t$-th iteration can be denoted as $\mathcal{Q}^{v}(t)=\left(\mathcal{Q}_{1}^{v}(t), \cdots, \mathcal{Q}_{k}^{v}(t), \cdots, \mathcal{Q}_{K}^{v}(t)\right)$ with quantization rate vector (at the $t$-th iteration) $\mathbf{L}^{v}(t)=$ $\left(L_{1}^{v}(t), \cdots, L_{K}^{v}(t)\right)$. Using the result (c) of Lemma 2 the TVCOQ design, which minimize $\tilde{E}_{\text {block }}^{\mathbf{w}}(\bar{T})$ under total quantization rate constraint over a horizon of $\bar{T}$ iterations, is equivalent to the following:

Problem 2 (TVCOQ Design Problem):

$$
\begin{aligned}
& \min _{\substack{\left\{\mathcal{Q}^{s}(t): 0 \leq t \leq \bar{T}\right\} \\
\text { or }\left\{\mathcal{Q}^{v}(t): 0 \leq t \leq \bar{T}\right\}}} \alpha^{\bar{T}-1} \sum_{t=0}^{\bar{T}-1} \alpha^{-t}\left\|\tilde{\mathbf{e}}_{t}\right\|_{\text {block }}^{\mathbf{w}} \\
& \text { s.t. } \sum_{t=0}^{\bar{T}-1} \sum_{m=1}^{n} L_{m}^{s}(t)=\bar{T} L, L_{m}^{s}(t) \in \mathbb{Z}^{+}(\forall m, t) \mathrm{SQ}
\end{aligned}
$$




$$
\text { or } \sum_{t=0}^{\bar{T}-1} \sum_{k=1}^{K} L_{k}^{v}(t)=\bar{T} L, L_{k}^{v}(t) \in \mathbb{Z}^{+}(\forall k, t) \mathrm{VQ}
$$

By introducing additional auxiliary variables $\{L(t)$ : $0 \leq t \leq \bar{T}-1\}$, where $L(t)$ can be interpreted as the per-stage sum quantization rate, and applying primal decomposition techniques, we can decompose Problem 2 into subproblems (per-stage TICOQ design $\mathcal{Q}^{s}(t)$ or $\left.\mathcal{Q}^{v}(t)(0 \leq t \leq \bar{T}-1)\right)$, which are given by

Problem 3: (TVCOQ Subproblems: Per-stage TICOQ Design Problem)

$$
\begin{aligned}
& \min _{\mathcal{Q}^{s}(t) \text { or } \mathcal{Q}^{v}(t)}\left\|\tilde{\mathbf{e}}_{t}(L(t))\right\|_{\text {block }}^{\mathbf{w}} \\
& \text { s.t. } \sum_{m=1}^{n} L_{m}^{s}(t)=L(t), L_{m}^{s}(t) \in \mathbb{Z}^{+}(\forall m) \mathrm{SQ} \\
& \text { or } \sum_{k=1}^{K} L_{k}^{v}(t)=L(t), L_{k}^{v}(t) \in \mathbb{Z}^{+}(\forall k) \mathrm{VQ}
\end{aligned}
$$

and the master problem (per-stage sum quantization rate $\{L(t): 0 \leq t \leq \bar{T}-1\}$ allocation among stages), which is given by

Problem 4: (TVCOQ Master Problem: Sum Quantization Rate Allocation Problem)

$$
\begin{aligned}
& \min _{\{L(t): 0 \leq t \leq \bar{T}-1\}} \alpha^{\bar{T}-1} \sum_{t=0}^{\bar{T}-1} \alpha^{-t}\left\|\tilde{\mathbf{e}}_{t}^{*}(L(t))\right\|_{\text {block }}^{\mathbf{w}} \\
& \text { s.t. } \sum_{t=0}^{\bar{T}-1} L(t)=\bar{T} L, L(t) \in \mathbb{Z}^{+}(0 \leq t \leq \bar{T}-1)
\end{aligned}
$$

where $\left\|\tilde{\mathbf{e}}_{t}^{*}(L(t))\right\|_{\text {block }}^{\mathbf{w}}$ is the optimal value of the $t$-th subproblem in Problem 3 for given $L(t)(0 \leq t \leq \bar{T}-1)$. Given the per-stage sum quantization rate $L(t)$, each $\mathrm{Per}$ stage TICOQ Design Problem in Problem 3 is the same as the TICOQ design problem in Section IV] and hence, the approaches in Section IV can be applied to solve Problem 3 for given $L(t)(0 \leq t \leq \bar{T}-1)$, including both SQ case and VQ case. In the following, we shall mainly discuss the Sum Quantization Rate $\{L(t): 0 \leq$ $t \leq \bar{T}-1\}$ Allocation in Problem 4 and analyze the tradeoff between convergence error and message passing overhead for the TVCOQ design.

\section{A. Time Varying Convergence-Optimal Scalar and Vec- tor Quantizers}

Similar to the TICOQ in Problem 1 each TVCOQ subproblem in Problem 3 is a Nonlinear Integer Programming (NLIP) problem. Brute-force solution to the TVCOQ master problem in Problem 4 requires exhaustive search, which is not acceptable. Therefore, we first apply continuous relaxation to the subproblems in Problem 3 to obtain the closed-form expression $\left\|\tilde{\mathbf{e}}_{t}^{*}\right\|_{\text {block }}^{\mathbf{w}}$ of the $t$-th subproblem. Based on the closed-form $\left\|\tilde{\mathbf{e}}_{t}^{*}\right\|_{\text {block }}^{\mathbf{w}}$, the master problem in Problem 4 becomes tractable, the solution of which is summarized in the following lemma.

Theorem 6: (Solution to TVCOQ Master Problem for SQ and VQ) For any given $\bar{T}$, assume $L \geq L^{\prime}-$ $n \frac{\bar{T}-1}{2} \log _{2} \alpha\left(L \in \mathbb{Z}^{+}\right)$. Let $\bar{L}^{*}(t)=n \log _{2}\left(\frac{\bar{\alpha}^{-t} \ln 2}{n \mu}\right)$, where $\mu>0$ is the LM of the constraint (32) chosen to satisfy the constraint $\sum_{t=0}^{\bar{T}-1} n \log _{2}\left(\frac{\alpha^{-t} \ln 2}{n \mu}\right)=\bar{T} L$. The optimal integer solution to the TVCOQ Master Problem in Problem 4 is given by

$$
L^{*}([t])= \begin{cases}\left\lceil\bar{L}^{*}([t])\right\rceil, & \text { if } t \leq \sum_{t=0}^{\bar{T}-1}\left(\bar{L}^{*}(t)-\left\lfloor\bar{L}^{*}(t)\right\rfloor\right) \\ \left\lfloor\bar{L}^{*}([t])\right\rfloor, & \text { otherwise }\end{cases}
$$

Proof: Please refer to Appendix G for the Proof.

Given the per-stage sum quantization rate $\left\{L^{*}(t)\right.$ : $0 \leq t \leq \bar{T}-1\}$ allocation obtained by Theorem 6 . the TVCOQ Subproblems in Problem 3 (similar to the TICOQ design problem in Section IV can be easily solved by Theorem 3 , 4 and 5.

\section{B. Tradeoff between Convergence Error and Message Passing Overhead}

In this subsection, we shall quantify the tradeoff between the error of the algorithm trajectory and the message passing overhead using TVCOQ. The following lemma summarizes the tradeoff results.

Lemma 6: (Performance Tradeoff of the Scalar and Vector TVCOQ) For any given $\bar{T}$, the convergence error bound (at the $\bar{T}$-th iteration) of the scalar and vector TVCOQ is given by $\tilde{E}_{\text {block }}^{\mathrm{w}}(\bar{T})=\bar{T} \alpha^{\frac{\bar{T}-1}{2}} \mathcal{O}\left(2^{-\frac{L}{n}}\right)$ for $L \geq L^{\prime}-n \frac{\bar{T}-1}{2} \log _{2} \alpha\left(L \in \mathbb{Z}^{+}\right)$, where $L^{\prime}$ is given by (24).

Proof: Please refer to Appendix G for the proof.

Remark 7: As $L \rightarrow \infty, \tilde{E}_{\text {block }}^{\mathbf{w}}(\bar{T}) \rightarrow 0$, which reduces to the conventional convergence results with perfect message passing. On the other hand, for any fixed $L$, as $\bar{T} \rightarrow \infty$, we have $\tilde{E}_{\text {block }}^{\mathbf{w}}(\bar{T}) \rightarrow 0$. Hence, using TVCOQ, one could achieve asymptotically zero convergence error even with finite $L$.

\section{Simulation Results and Discussions}

In this section, we shall evaluate the performance of the proposed time invariant convergence-optimal quantizer (TICOQ) and time varying convergence-optimal quantizer (TVCOQ) for the contracting iterations by simulations. We consider distributed precoding updates in MIMO interference game with $K$ transmitter and receiver pairs where the transmit convariance matrix $\mathbf{P}_{k}$ of the $k$-th transmitter is iteratively updated (and quantized) according to 15 . In the simulation, we consider $K=2,4,8$ noncooperative transmitter-receiver pairs with $N=2,4$ transmit/receive antenna. The distance 


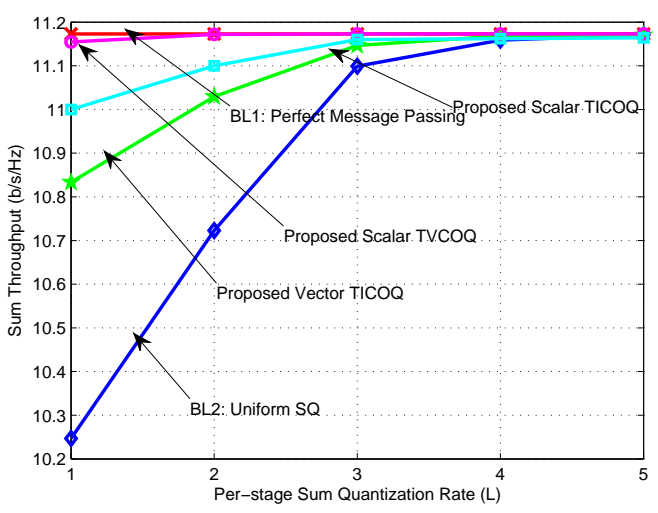

(a) Sum throughput

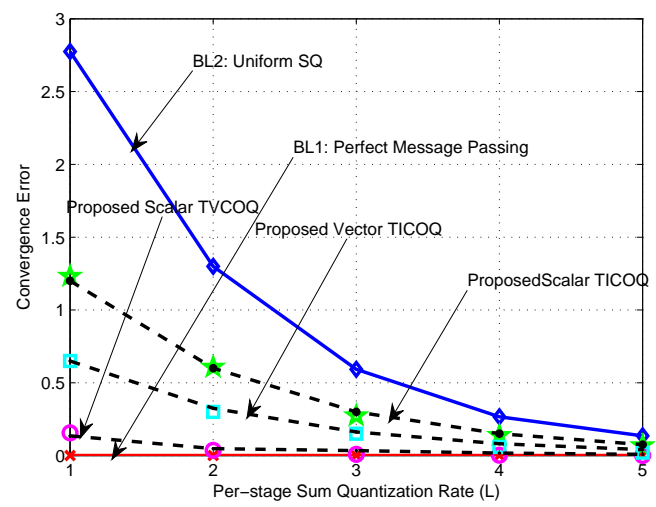

(b) Convergence error

Fig. 2. Sum throughput/convergence error versus per-stage sum quantization rate $L$ (bits) of 2 pairs MIMO interference game with 2 transmit and receive antennas, $d_{11}=d_{22}=100 \mathrm{~m}, d_{12}=200$ $\mathrm{m}, d_{21}=500 \mathrm{~m}$, path loss exponent $\gamma=3.5$, and transmit power of $P_{1}=P_{2}=10 \mathrm{dBm}$. The total number of iterations is $\bar{T}=4$ and the per-stage quantization rate per antenna is $\frac{L}{K \times N^{2}}=\frac{L}{8}$. In (b), the "*", "o", etc represent the simulation results of the proposed TICOQ and TVCOQ, while the dashed line represents the analytical expression $\mathcal{O}\left(2^{-\frac{L}{n}}\right)$ (TICOQ) and $\bar{T} \alpha^{\frac{\bar{T}-1}{2}} \mathcal{O}\left(2^{-\frac{L}{n}}\right)$ at fixed $\bar{T}=4$ (TVCOQ).

from the $k$-th transmitter to the $j$-th receiver is denoted as $d_{k j}$. The bandwidth is $10 \mathrm{MHZ}$. The pathloss exponent is $\gamma=3.5$. Each element of the small scale fading channel matrix is $\mathcal{C N}(0,1)$ distributed. We compare the performance with two reference baselines. Baseline 1 (BL1) refers to the case with perfect message passing [5]. Baseline 2 (BL2) refers to the case with uniform scalar quantizer.

\section{A. Performance of the TICOQ}

Fig. 2 (a) and Fig. 2 (b) illustrate the sum throughput and convergence error (w.r.t. the weighted blockmaximum norm $\|\cdot\|_{\text {block }}^{\mathbf{w}}$ ) versus the per-stage sum quantization rate of 2 pairs MIMO interference game under fixed number of iterations. The sum throughput

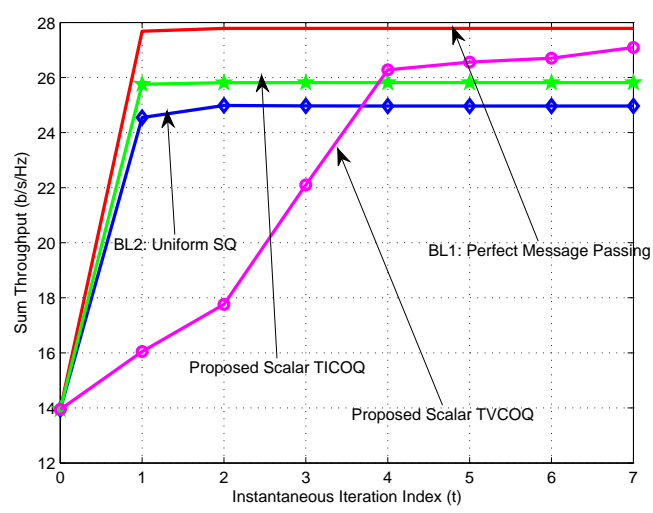

(a) Sum throughput

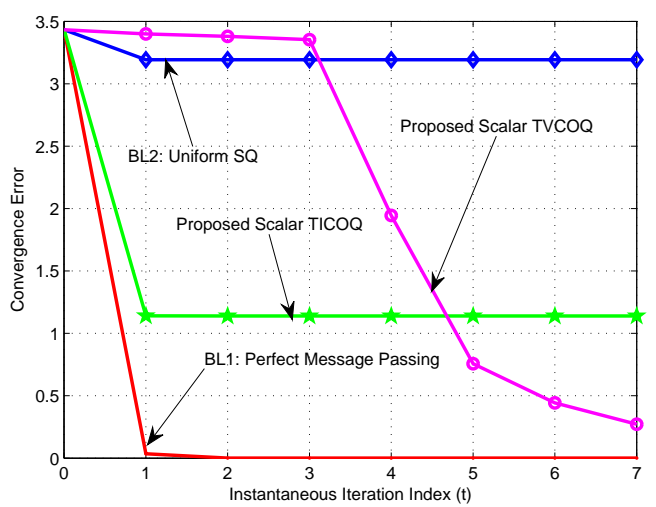

(b) Convergence error

Fig. 3. Sum throughput/convergence error versus instantaneous iteration index $t$ of 4 pairs MIMO interference game with 4 transmit and receive antennas, $d_{i i}=100 \mathrm{~m}, d_{i j}=200 \mathrm{~m}(i<j), d_{i j}=500$ $\mathrm{m}(i>j)$, path loss exponent $\gamma=3.5$, and transmit power of $P_{1}=P_{2}=5 \mathrm{dBm}$. The per-stage sum quantization rate $L=64$ bits (i.e. the per-stage quantization rate per antenna is $\frac{L}{K \times N^{2}}=\frac{L}{64}=1$ bit), and the total number of iterations $\bar{T}=7$.

of all the schemes increases as $L$ decreases due to the decreasing convergence error. It can be observed that the proposed scalar and vector TICOQ have significant performance gain in sum throughput and convergence error compared with the commonly used uniform scalar quantizer. In addition, the vector TICOQ outperforms the scalar TICOQ in convergence performance at the cost of the higher encoding and decoding complexity.

Fig. 3 and Fig. 4 show the sum throughput and convergence error versus the instantaneous iteration time index of MIMO interference game under a fixed perstage sum quantization rate and total number of iterations with different $K$ and $N$. It can be seen that in all cases, the proposed scalar and vector TICOQ have significant performance gain in sum throughput and convergence error compared with the commonly used uniform scalar qunatizer. 


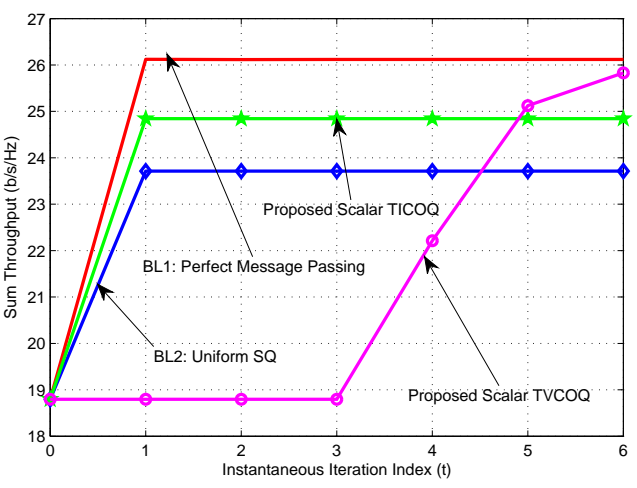

(a) Sum throughput

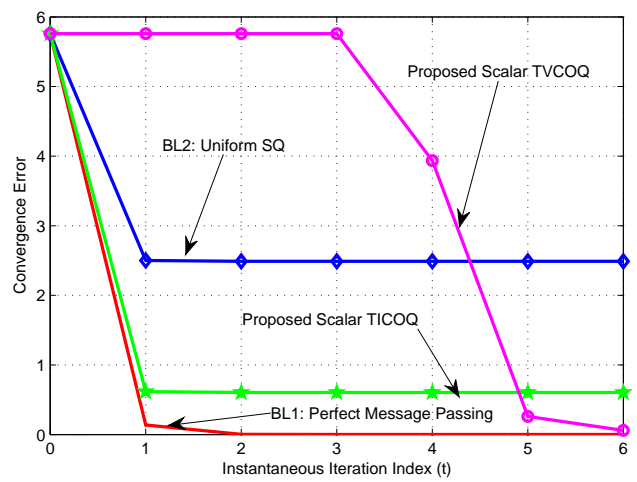

(b) Convergence error

Fig. 4. Sum throughput/convergence error versus instantaneous iteration index $t$ of 8 pairs MIMO interference game with 2 transmit and receive antennas, $d_{i i}=100 \mathrm{~m}, d_{i j}=200 \mathrm{~m}(i<j), d_{i j}=500$ $\mathrm{m}(i>j)$, path loss exponent $\gamma=3.5$, and transmit power of $P_{1}=P_{2}=5 \mathrm{dBm}$. The per-stage sum quantization rate $L=32$ bits (i.e. the per-stage quantization rate per antenna is $\frac{L}{K \times N^{2}}=\frac{L}{32}=1$ bit), and the total number of iterations $\bar{T}=6$.

\section{B. Performance of the TVCOQ}

From Fig. 2 (a) and Fig. 2 (b), we can observe that the proposed TVCOQ has significant performance gain in sum throughput and convergence error compared with commonly used uniform scalar qunatizer and TICOQ. For example, the sum throughput of the TVCOQ is very close to that with perfect message passing. Fig. 3 and 4 illustrate the transient performance of the TVCOQ versus iteration index $t$. We observe that the performance of the TVCOQ improves as $t$ increases. This is because the TVCOQ optimizes the quantization rate allocation over both the node domain and the time domain (over a horizon of $\bar{T}$ iterations).

\section{Tradeoff between Convergence Error and Message Passing Overhead}

Fig. 2 (b) illustrates the tradeoff between convergence error and message passing overhead (in terms of $L$ at

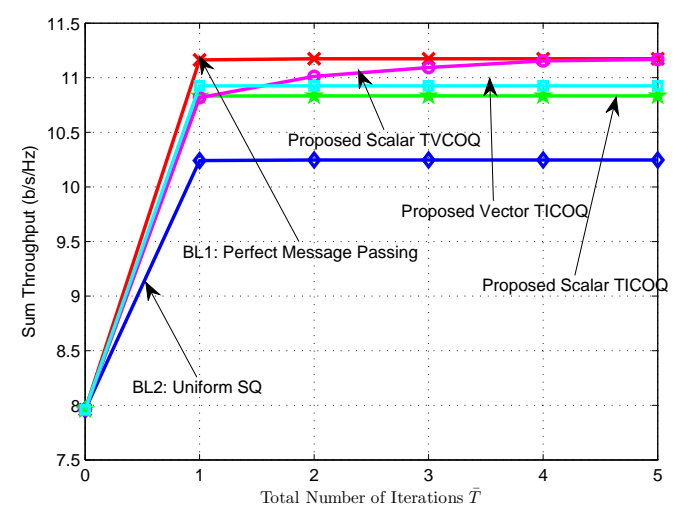

(a) Sum throughput

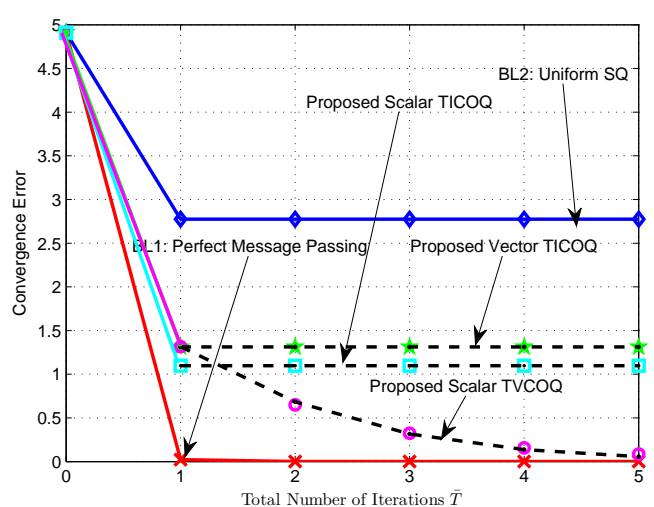

(b) Convergence error

Fig. 5. Sum throughput/convergence error versus the total number of iterations $\bar{T}$ of 2 pairs MIMO interference game with 2 transmit and receive antennas, $d_{11}=d_{22}=100 \mathrm{~m}, d_{12}=200 \mathrm{~m}, d_{21}=500 \mathrm{~m}$, path loss exponent $\gamma=3.5$, and transmit power of $P_{1}=P_{2}=10$ $\mathrm{dBm}$. The per-stage sum quantization rate $L=8$ bits (i.e. the per-stage quantization rate per antenna is $\frac{L}{K \times N^{2}}=\frac{L}{8}=1$ bit). In (b), the "**, "o", etc represent the simulation results of the proposed TICOQ and TVCOQ, while the dashed line represents the analytical expression $\mathcal{O}\left(2^{-\frac{L}{n}}\right)$ (TICOQ) and $\bar{T} \alpha^{\frac{\bar{T}-1}{2}} \mathcal{O}\left(2^{-\frac{L}{n}}\right)$, with $\bar{T} \geq 1$ (TVCOQ) at fixed $L=8$ bits.

fixed total number of iterations $\bar{T}$ ). As the message passing overhead $L$ increases, the convergence error of all the proposed quantization schemes approaches to 0 with order $\mathcal{O}\left(2^{-\frac{L}{n}}\right)$ under fixed $\bar{T}$, which verified the results in Lemma 5 and 6 . Similarly, the sum throughput of all the schemes increases as $L$ decreases due to the decreasing convergence error as shown in Fig. 2 (a).

Fig. 5 (b) shows the tradeoff between convergence error and message passing overhead (in terms of $\bar{T}$ at fixed $L$ ). As the total number of iterations increases, the convergence error of TVCOQ decreases, while the convergence error of the TICOQ and the uniform quantizer fail to decrease. It is because that TICOQ has steady state convergence error floor for any finite $L$ (i.e. $\bar{E}_{\text {block }}^{\mathbf{w}}(\infty)=\frac{1}{1-\alpha} \mathcal{O}\left(2^{-\frac{L}{n}}\right)>0$ shown in Lemma 5], 
while the convergence error of TVCOQ goes to 0 as $\bar{T}$ goes to infinity (i.e. $\tilde{E}_{\text {block }}^{\mathrm{w}}(\bar{T})=\bar{T} \alpha^{\frac{\bar{T}-1}{2}} \mathcal{O}\left(2^{-\frac{L}{n}}\right) \rightarrow 0$ as $\bar{T} \rightarrow \infty$ shown in Lemma [6). Similarly, as the total number of iterations increases, the sum throughput performance of the TVCOQ improves but this is not the case for TICOQ and the Baseline 2 as shown in Fig. 5 (a).

\section{SUMMARY}

In this paper, we study the convergence behavior of general iterative function evaluation algorithms with quantized message passing. We first obtain closed-form expressions of the convergence performance under quantized message passing among distributed nodes. To minimize the effect of the quantization error on the convergence, we propose the time invariant convergenceoptimal quantizer (TICOQ) and the time varying convergence-optimal quantizer (TVCOQ). We found that the convergence error scales with the number of bits for quantized message passing in the order of $\frac{1}{1-\alpha} \mathcal{O}\left(2^{-\frac{L}{n}}\right)$ and $\bar{T} \alpha^{\frac{\bar{T}-1}{2}} \mathcal{O}\left(2^{-\frac{L}{n}}\right)$ for TICOQ and TVCOQ respectively. Finally, we illustrate using MIMO interference game as example that the proposed designs achieve significant gain in the convergence performance.

\section{APPENDIX}

\section{Appendix A: PROOF of LEMma 1}

First, we prove conclusion (a). By the update equation in (14), the triangle inequality of norm and the property of the contraction mapping, we have $\left\|\mathbf{x}(t)-\mathrm{x}^{*}\right\|=$ $\left\|\mathbf{T}(\mathbf{x}(t-1))+\mathbf{e}(t-1)-\mathbf{x}^{*}\right\| \leq \| \mathbf{T}(\mathbf{x}(t-1))-$ $\mathbf{x}^{*}\|+\| \mathbf{e}(t-1)\|\leq \alpha\| \mathbf{x}(t-1)-\mathbf{x}^{*}\|+\| \mathbf{e}(t-1) \|=$ $\alpha\left\|\mathbf{T}(\mathbf{x}(t-2))+\mathbf{e}(t-2)-\mathbf{x}^{*}\right\|+\|\mathbf{e}(t-1)\| \leq \cdots \leq$ $\alpha^{t}\left\|\mathbf{x}(0)-\mathbf{x}^{*}\right\|+E(t)$, where $E(t) \triangleq \sum_{l=1}^{t} \alpha^{l-1} \| \mathbf{e}(t-$ $l)\left\|\stackrel{(1)}{=} \sum_{l^{\prime}=0}^{l-1} \alpha^{t-l^{\prime}-1}\right\| \mathbf{e}\left(l^{\prime}\right)\left\|\stackrel{(2)}{=} \alpha^{t-1} \sum_{l=0}^{t-1} \alpha^{-l}\right\| \mathbf{e}(l) \|$, (1) is obtained by denoting $l^{\prime}=t-l$, and (2) is obtained by denoting $l=l^{\prime}$. (b) is trivial. Finally, we prove conclusion (c). Since $\|\mathbf{e}(t)\| \leq\|\overline{\mathbf{e}}\| \forall t$, we have $E(t)=$ $\sum_{l=1}^{t} \alpha^{l-1}\|\mathbf{e}(t-l)\| \leq \bar{E}(t) \triangleq \sum_{l=1}^{t} \alpha^{l-1}\|\overline{\mathbf{e}}\|=$ $\frac{1-\alpha^{t}}{1-\alpha}\|\overline{\mathbf{e}}\|$ and $\bar{E}(\infty) \triangleq \lim _{t \rightarrow \infty} \bar{E}(t)=\frac{\overline{\|} \mathbf{\overline { \mathbf { e } }} \|}{1-\alpha}$. Given the limiting error bound $\bar{E}(\infty)$, we know that $\exists T$ s.t. $\forall t>$ $T, \mathbf{x}(t) \in \mathbb{S}$. If $\mathbf{x}=\mathcal{Q}(\mathbf{T}(\mathbf{x})) \forall \mathbf{x} \in \mathbb{S}$, then $\mathbf{x}(t) \rightarrow$ $\mathbf{x}(\infty) \in \mathbb{S}$. Thus, we obtain the sufficient condition for convergence. On the other hand, if $\mathbf{x} \neq \mathcal{Q}(\mathbf{T}(\mathbf{x}))$ $\forall \mathbf{x} \in \mathbb{S}, \mathbf{x}(t)$ will not converge, but jumps among (at least two) points in $\mathbb{S}$. Thus, we obtain the necessary condition for convergence.

\section{Appendix B: Proof of Lemma 2}

Jacobi scheme in (16) shares the similar form as 114. Therefore, the proof of Jacobi scheme is the same as that in Appendix A, except based on weighted blockmaximum norm.

Next, we prove the convergence for the Gauss-Seidel scheme under quantized message passing. Let $\hat{\mathbf{x}}^{k}=\mathbf{x}$ for $k=1$ and $\hat{\mathbf{x}}^{k}=\left(\hat{\mathbf{S}}_{1}(\mathbf{x})+\mathbf{e}_{1}, \cdots, \hat{\mathbf{S}}_{k-1}(\mathbf{x})+\right.$ $\left.\mathbf{e}_{k-1}, \mathbf{x}_{k}, \cdots, \mathbf{x}_{K}\right)$ for $2 \leq k \leq K$. By the definition of weighted block-maximum norm and the property of block-contraction $\mathbf{T}$, we have

$$
\begin{aligned}
& \frac{\left\|\hat{\mathbf{S}}_{k}(\mathbf{x})-\mathbf{x}_{k}^{*}\right\|_{k}}{w_{k}}=\frac{\left\|\mathbf{T}_{k}\left(\hat{\mathbf{x}}^{k}\right)-\mathbf{T}_{k}\left(\mathbf{x}^{*}\right)\right\|_{k}}{w_{k}} \\
& \leq\left\|\mathbf{T}\left(\hat{\mathbf{x}}^{k}\right)-\mathbf{T}\left(\mathbf{x}^{*}\right)\right\|_{\text {block }}^{\mathbf{w}} \leq \alpha\left\|\hat{\mathbf{x}}^{k}-\mathbf{x}^{*}\right\|_{\text {block }}^{\mathbf{w}} \\
& \left\{\begin{array}{cl}
\leq \alpha\left\|\mathbf{x}-\mathbf{x}^{*}\right\|_{\text {block }}^{\mathbf{w}}, & k=1 \\
=\alpha \max \left\{\max _{j<k} \frac{\left\|\hat{\mathbf{S}}_{j}(\mathbf{x})+\mathbf{e}_{j}-\mathbf{x}_{j}^{*}\right\|_{j}}{w_{j}},\right. & \\
\left.\max _{j \geq k} \frac{\left\|\mathbf{x}_{j}-\mathbf{x}_{j}^{*}\right\|_{j}}{w_{j}}\right\}, & 2 \leq k \leq K
\end{array}\right.
\end{aligned}
$$

When $k=2$, by (34), we have

$$
\begin{aligned}
& \frac{\left\|\hat{\mathbf{S}}_{2}(\mathbf{x})-\mathbf{x}_{2}^{*}\right\|_{2}}{w_{2}} \\
\leq & \alpha \max \left\{\frac{\left\|\hat{\mathbf{S}}_{1}(\mathbf{x})+\mathbf{e}_{1}-\mathbf{x}_{1}^{*}\right\|_{1}}{w_{1}}, \max _{j \geq 2} \frac{\left\|\mathbf{x}_{j}-\mathbf{x}_{j}^{*}\right\|_{j}}{w_{j}}\right\} \\
\leq & \alpha \max \left\{\frac{\left\|\hat{\mathbf{S}}_{1}(\mathbf{x})-\mathbf{x}_{1}^{*}\right\|_{1}}{w_{1}}+\frac{\left\|\mathbf{e}_{1}\right\|_{1}}{w_{1}}, \max _{j \geq 2} \frac{\left\|\mathbf{x}_{j}-\mathbf{x}_{j}^{*}\right\|_{j}}{w_{j}}\right\} \\
\leq & \alpha\left\|\mathbf{x}-\mathbf{x}^{*}\right\|_{\text {block }}^{\mathbf{w}}+\alpha\|\mathbf{e}\|_{\text {block }}^{\mathbf{w}} \stackrel{\text { by iteration }}{\Rightarrow} \\
& \frac{\left\|\hat{\mathbf{S}}_{k}(\mathbf{x})-\mathbf{x}_{k}^{*}\right\|_{k}}{w_{k}} \leq \alpha\left\|\mathbf{x}-\mathbf{x}^{*}\right\|_{\text {block }}^{\mathbf{w}}+\sum_{l=1}^{k-1} \alpha^{l}\|\mathbf{e}\|_{\text {block }}^{\mathbf{w}}, \forall k \\
\Rightarrow & \left\|\hat{\mathbf{S}}(\mathbf{x})-\mathbf{x}^{*}\right\|_{\text {block }}^{\mathbf{w}} \leq \alpha\left\|\mathbf{x}-\mathbf{x}^{*}\right\|_{\text {block }}^{\mathbf{w}} \\
\Rightarrow & \left\|\mathbf{x}(t)-\mathbf{x}^{*}\right\|_{\text {block }}^{\mathbf{w}} \quad+\frac{\alpha\left(1-\alpha^{K-1}\right)}{1-\alpha}\|\mathbf{e}\|_{\text {block }}^{\mathbf{w}} \\
= & \left\|\hat{\mathbf{S}}(\mathbf{x}(t-1))+\mathbf{e}(t-1)-\mathbf{x}^{*}\right\|_{\text {block }}^{\mathbf{w}} \\
\leq & \alpha{ }^{t}\left\|\mathbf{x}(0)-\mathbf{x}^{*}\right\|_{\text {block }}^{\mathbf{w}}+E_{\text {block }}^{\mathbf{w}}(t)
\end{aligned}
$$

where $E_{\text {block }}^{\mathbf{w}}(t) \triangleq \frac{1-\alpha^{K}}{1-\alpha} \alpha^{t-1} \sum_{l=0}^{t-1} \alpha^{-l}\|\mathbf{e}(l)\|_{\text {block }}^{\mathbf{w}}$. Since we have shown $\left\|\mathbf{x}(t)-\mathbf{x}^{*}\right\|_{\text {block }}^{\mathbf{w}} \leq \alpha^{t} \| \mathbf{x}(0)-$ $\mathbf{x}^{*} \|_{\text {block }}^{\mathbf{w}}+E_{\text {block }}^{\mathbf{w}}(t)$, which is the same as the conclusion in Lemma 1 (a) except for the different norm $\|\cdot\|_{\text {block }}^{\mathbf{w}}$ and the extra scalar $\frac{1-\alpha^{K}}{1-\alpha}>1$ (indicating the additional error due to the incremental nature of the Gauss-Seidel update) in $E_{\mathrm{block}}^{\mathbf{w}}(t)$, we can follow the similar steps in Appendix A to obtain the conclusion for Gauss-Seidel scheme.

\section{Appendix C: Proof of Lemma 3}

First, we shall show that $\|\cdot\|_{\text {block }}^{\mathbf{w}}$ is monotone (absolute). Denote $|\mathbf{x}| \triangleq\left(\left|x_{1}\right|, \cdots,\left|x_{n}\right|\right)$. We say that $|\mathbf{x}| \leq|\mathbf{y}|$ if $\left|x_{m}\right| \leq\left|y_{m}\right| \forall m$. Due to the monotonicity of $\|\cdot\|_{k}(\forall k)$, we have $|\mathbf{x}| \leq|\mathbf{y}| \Leftrightarrow\left|\mathbf{x}_{k}\right| \leq\left|\mathbf{y}_{k}\right|(\forall k) \Rightarrow$ 
$\left\|\mathbf{x}_{k}\right\|_{k} \leq\left\|\mathbf{y}_{k}\right\|_{k}(\forall k) \Rightarrow \max _{k} \frac{\left\|\mathbf{x}_{k}\right\|_{k}}{w_{k}} \leq \max _{k} \frac{\left\|\mathbf{y}_{k}\right\|_{k}}{w_{k}} \Leftrightarrow$ $\|\mathbf{x}\|_{\text {block }}^{\mathbf{w}} \leq\|\mathbf{y}\|_{\text {block }}^{\mathbf{w}}$. Thus, $\|\cdot\|_{\text {block }}^{\mathbf{w}}$ is monotone (absolute). Next, we shall show that each coordinate scalar TICOQ is uniform quantizer. Given any $\mathbf{L}^{s}$ s.t. 20 is satisfied, by the monotonicity of $\|\cdot\|_{\text {block }}^{\mathbf{w}}$, we can easily prove $\min _{\mathcal{Q}^{s}\left(\mathbf{L}^{s}\right)}\|\overline{\mathbf{e}}\|_{\text {block }}^{\mathbf{w}} \Leftrightarrow \min _{\mathcal{Q}_{m}^{s}\left(L_{m}^{s}\right)} \bar{e}_{m}(\forall m)$. In other words, given any $L_{m}^{s}$, the coordinate scalar TICOQ $\mathcal{Q}_{m}^{s *}\left(L_{m}^{s}\right)$ should minimize the worst-case error $\left|\bar{e}_{m}\right|$ for the $m$-th coordinate of the input vector. Since the uniform quantizer minimizes the worst-case error regardless of the shape of the input pdf [20], each coordinate scalar TICOQ $\mathcal{Q}_{m}^{s *}$ is a uniform quantizer.

\section{Appendix D: Proof of Theorem 3 And Theorem 4}

When $\|\cdot\|_{k}$ is weighted maximum norm, the objective function becomes $\|\overline{\mathbf{e}}\|_{\text {block }}^{\mathbf{w}}=\max _{k} \frac{\left\|\overline{\mathbf{e}}_{k}\right\|_{k}}{w_{k}}=$ $\max _{k} \frac{\max _{m \in \mathcal{M}_{k}} \frac{\left|\bar{e}_{m}\right|}{a_{m}}}{w_{k}}=\max _{m} \frac{\left|\bar{e}_{m}\right|}{a_{m}\left(\sum_{k=1}^{K} w_{k} \mathbf{I}\left[m \in \mathcal{M}_{k}\right]\right)}=$ $\max _{m} C_{m} 2^{-L_{m}^{s}}$. Therefore, we have $\min _{\mathcal{Q}^{s}}\|\overline{\mathbf{e}}\|_{\text {block }}^{\mathbf{w}}=$ $\min _{\mathbf{L}^{s}}\left(\max _{m} C_{m} 2^{-L_{m}^{s}}\right)$. By continuous relaxation and equivalent transformation of minimax problems [25], the minimax problem in Problem 1 is equivalent to the following problem (under continuous relaxation), which is in epigraph form with optimization variables $\left\{\bar{L}_{m}^{s}\right\}, \tau$ :

$$
\begin{aligned}
\left(\bar{P}^{s}\right): \min _{\left\{\bar{L}_{m}^{s}\right\}, \tau} \tau & \\
\text { s.t. } & C_{m} 2^{-\bar{L}_{m}^{s}} \leq \tau(1 \leq m \leq n) \\
& \sum_{m=1}^{n} \bar{L}_{m}^{s}=L, \bar{L}_{m}^{s} \geq 0(1 \leq m \leq n)
\end{aligned}
$$

$\left(\bar{P}^{s}\right)$ is a convex optimization problem. It can be easily shown that the Slater's condition holds. Therefore, we shall get the optimal solution to the relaxed problem through KKT conditions. The Lagrangian of $\left(\bar{P}^{s}\right)$ is given by $\mathcal{L}^{s}\left(\overline{\mathbf{L}}^{s}, \tau, \boldsymbol{\lambda}^{s}, \boldsymbol{\nu}^{s}, \mu^{s}\right)=$ $\tau+\sum_{m=1}^{n} \lambda_{m}^{s}\left(C_{m} 2^{-\bar{L}_{m}^{s}}-\tau\right)-\sum_{m=1}^{n} \nu_{m}^{s} \bar{L}_{m}^{s}+$ $\mu^{s}\left(\sum_{m=1}^{n} \bar{L}_{m}^{s}-L\right)$, where $\boldsymbol{\lambda}^{s}, \boldsymbol{\nu}^{s}, \mu^{s}$ are the Lagrangian multipliers (LM). $\overline{\mathbf{L}}^{s}, \tau, \boldsymbol{\lambda}^{s}, \boldsymbol{\nu}^{s}, \mu^{s}$ are optimal iff they satisfy the following KKT conditions: (a) primal constraints: (36), 35); (b) dual constraints: $\lambda^{s} \succeq$ $0, \boldsymbol{\nu}^{s} \succeq 0$; (c) complementary slackness: $\lambda_{m}^{s}\left(C_{m} 2^{-\bar{L}_{m}^{s}-}\right.$ $\tau)=0(\forall m), \quad \nu_{m}^{s} \bar{L}_{m}^{s}=0(\forall m) ; \quad(\mathrm{d}) \frac{\partial \mathcal{L}^{s}}{\partial L_{m}^{s}}=$ $-\ln (2) \lambda_{m}^{s} C_{m} 2^{-\bar{L}_{m}^{s}}-\nu_{m}^{s}+\mu^{s}=0(\forall m), \frac{\partial \mathcal{L}^{s}}{\partial \tau}=$ $1-\sum_{m=1}^{n} \lambda_{m}^{s}=0$. Thus, $\forall m$, we have

$$
\begin{aligned}
& \text { if } \frac{\ln (2) \lambda_{m}^{s} C_{m}}{\mu^{s}}>1: \lambda_{m}^{s}=\frac{\mu^{s}}{\ln (2) \tau}, \nu_{m}^{s}=0, \\
& \bar{L}_{m}^{s *}=\log _{2}\left(\frac{\ln (2) \lambda_{m}^{s} C_{m}}{\mu^{s}}\right), \tau=\frac{\mu^{s}}{\ln (2) \lambda_{m}^{s}}, \mu^{s}>0 \\
& \text { if } \frac{\ln (2) \lambda_{m}^{s} C_{m}}{\mu^{s}} \leq 1: \lambda_{m}^{s}=0, \nu_{m}^{s}=\mu^{s}, \bar{L}_{m}^{s *}=0, \mu^{s}>0
\end{aligned}
$$

where LMs $\left\{\lambda_{m}^{s}\right\}, \mu^{s}$ are chosen to satisfy $\sum_{m=1}^{n}\left(\log _{2} \frac{\ln (2) \lambda_{m}^{s} C_{m}}{\mu^{s}}\right)^{+}=L$ and $1-\sum_{m=1}^{n} \lambda_{m}^{s}=0$. Substitute $\tau=\frac{\mu^{s}}{\ln (2) \lambda_{m}^{s}}$ into $\bar{L}_{m}^{s *}$, we have $\bar{L}_{m}^{s *}=\left(\log _{2} \frac{C_{m}}{\tau}\right)^{+}$, where $\tau$ is chosen to satisfy $\sum_{m=1}^{n}\left(\log _{2} \frac{C_{m}}{\tau}\right)^{+}=L$. Furthermore, substituting the relaxed solution $\bar{L}_{m}^{s *}$ into the transformed problem $\left(\bar{P}^{s}\right)$, the optimal value of $\left(\bar{P}^{s}\right)$ is given by $\tau$ and this is also the optimal value $\left\|\overline{\mathbf{e}}^{*}\right\|_{\text {block }}^{\mathbf{w}}$ of the original optimization Problem 1 (under continuous relaxation) due to the equivalence of the epigraph transformation. Next, we are trying to prove that the rounding strategy in (22) in Theorem 3 is the optimal integer solution of Problem 11 Suppose we round $\bar{L}_{m}^{s *}$ to $\left\lfloor\bar{L}_{m}^{s *}\right\rfloor$ and let $\delta_{m}^{s}=\bar{L}_{m}^{s *}-\left\lfloor\bar{L}_{m}^{s *}\right\rfloor$. Denote $b_{m}=C_{m} 2^{-\left\lfloor\bar{L}_{m}^{s *}\right\rfloor}=C_{m} 2^{-\left(\bar{L}_{m}^{s *}-\delta_{m}^{s}\right)}$, i.e. $b_{m}=C_{m}$ if $C_{m} \leq \tau$ and $b_{m}=2^{\delta_{m}^{s}} \tau$ otherwise. The value of the objective function in Problem 1 is $\max _{m} b_{m}$. Reducing $\bar{L}_{m}^{s *}$ by $N+\delta_{m}^{s}\left(N \geq 1, N \in \mathbb{Z}^{+}\right)$ $\forall m$ for integer solution will lead to the value of objective function greater than $\max _{m} b_{m}$. On the other hand, since the optimal value of the original integer programming problem is greater than the optimal value under continuous relaxation, increasing $\bar{L}_{m}^{s *}$ by $N-\delta_{m}^{s}\left(N>1, N \in \mathbb{Z}^{+}\right) \forall m$ will not help further reducing the value of the objective function. Therefore, the optimal integer solution $\left\{L_{m}^{s *}\right\}$ satisfies $\delta_{m}^{s}-1 \leq \bar{L}_{m}^{s *}-L_{m}^{s *} \leq \delta_{m}^{s}$ and the rounding strategy in (22) is the optimal integer solution of Problem 1

When $\|\cdot\|_{k}$ is $L_{p}$ norm, the objective function becomes $\|\overline{\mathbf{e}}\|_{\text {block }}^{\mathbf{w}}=\max _{k} \frac{\left\|\overline{\mathbf{e}}_{k}\right\|_{k}}{\left.w_{k} \bar{e}_{m}\right|^{p}}=\max _{k} \frac{\left(\sum_{m \in \mathcal{M}_{k}}\left|\bar{e}_{m}\right|^{p}\right)^{\frac{1}{p}}}{w_{k}}=$ $\max _{k}\left(\sum_{m \in \mathcal{M}_{k}} \frac{\left|\bar{e}_{m}\right|^{p}}{\sum_{k=1}^{K} w_{k}^{p} \mathbf{I}\left[m \in \mathcal{M}_{k}\right]}\right)^{\frac{1}{p}}$

$\max _{k}\left(\sum_{m \in \mathcal{M}_{k}} C_{m} 2^{-p L_{m}^{s}}\right)^{\frac{1}{p}}$ we have $\min _{\mathcal{Q}^{s}}\|\overline{\mathbf{e}}\|_{\text {block }}^{\mathbf{w}}=$ $\min _{\mathbf{L}^{s}}\left(\max _{k}\left(\sum_{m \in \mathcal{M}_{k}} C_{m} 2^{-p L_{m}}\right)^{\frac{1}{p}}\right)$. Using similar of minimax problems [25], the minimax problem in Problem 1 is equivalent (under continuous relaxation) to the following problem, which is in epigraph form with optimization variables $\left\{\bar{L}_{m}^{s}\right\}, \tau$ :

$$
\begin{aligned}
\left(\bar{Q}^{s}\right): & \min _{\bar{L}_{m}^{s}, \tau} \tau \\
& \text { s.t. } \sum_{m \in \mathcal{M}_{k}} C_{m} 2^{-p \bar{L}_{m}^{s}} \leq \tau(1 \leq k \leq K)
\end{aligned}
$$

constraint in 36

$\left(\bar{Q}^{s}\right)$ is a convex optimization problem. Using similar argument as in the weighted maximum norm case, the Lagrangian of $\left(\bar{Q}^{s}\right)$ is given by $\mathcal{L}^{s}\left(\overline{\mathbf{L}}^{s}, \tau, \boldsymbol{\lambda}^{s}, \boldsymbol{\nu}^{s}, \mu^{s}\right)=$

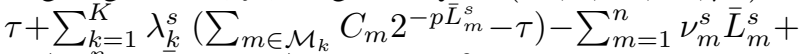
$\mu^{s}\left(\sum_{m=1}^{n} \bar{L}_{m}^{s}-L\right)$, where $\boldsymbol{\lambda}^{s}, \boldsymbol{\nu}^{s}, \mu^{s}$ are the LMs. Using standard KKT conditions, the optimal solution of $\left(\bar{Q}^{s}\right)$ is $\bar{L}_{m}^{s *}=\frac{1}{p} \log _{2}\left(\frac{p \ln (2)\left(\sum_{k=1}^{K} \lambda_{k}^{s} \mathbf{1}\left[m \in \mathcal{M}_{k}\right]\right) C_{m}}{\mu^{s}} \vee\right.$ 
1) and $\sum_{m \in \mathcal{M}_{k}} C_{m} 2^{-p \bar{L}_{m}^{s}}=\sum_{m \in \mathcal{M}_{k}}\left(\frac{\mu^{s}}{p \ln (2) \lambda_{k}^{s}} \wedge\right.$ $\left.C_{m}\right)=\tau$ if $\lambda_{k}^{s}>0, x \vee a \triangleq \max \{x, a\}, x \wedge a \triangleq$ $\min \{x, a\}$, where the LMs $\left\{\lambda_{k}^{s}\right\}, \mu^{s}$ are chosen to satisfy $\sum_{m=1}^{n} \frac{1}{p} \log _{2}\left(\frac{p \ln (2)\left(\sum_{k=1}^{K} \lambda_{k}^{s} \mathbf{1}\left[m \in \mathcal{M}_{k}\right]\right) C_{m}}{\mu^{s}} \vee 1\right)=L$ and $\sum_{k=1}^{K} \lambda_{k}^{s}=1$. Let $\tau_{k}=\frac{\mu^{s}}{p \ln (2) \lambda_{k}^{s}}$, then we have $\bar{L}_{m}^{s *}=\frac{1}{p} \log _{2}\left(\frac{C_{m}}{\sum_{k=1}^{K} \tau_{k} \mathbf{I}\left[m \in \mathcal{M}_{k}\right]} \vee 1\right)$, where $\left\{\tau_{k}\right\}$ and $\tau$ are constants related to the LMs $\left\{\lambda_{k}^{s}\right\}, \mu^{s}$ of the constraints (36), (37) in problem $\left(\bar{Q}^{s}\right)$. They are chosen to satisfy $\sum_{k=1}^{K} \sum_{m \in \mathcal{M}_{k}} \frac{1}{p} \log _{2}\left(\frac{C_{m}}{\tau_{k}} \vee 1\right)=L$ and $\frac{1}{\tau_{k}}\left(\sum_{m \in \mathcal{M}_{k}}\left(C_{m} \wedge \tau_{k}\right)-\tau\right)=0(\forall k)$. Finally, substituting $\bar{L}_{m}^{s *}$ into the transformed problem $\left(\bar{Q}^{s}\right)$, the optimal value of $\left(\bar{Q}^{s}\right)$ is given by $\tau^{\frac{1}{p}}$ and this is also the optimal value $\left\|\overline{\mathbf{e}}^{*}\right\|_{\text {block }}^{\mathbf{w}}$ of the original optimization Problem 1 (under continuous relaxation) due to the equivalence of the epigraph transformation.

\section{APPEndix E: ProOF OF LEMma 4 AND 5}

Since $\left\|\mathbf{x}_{k}\right\|_{k} \leq\left\|\mathbf{y}_{k}\right\|_{k}(\forall k) \Rightarrow\|\mathbf{x}\|_{\text {block }}^{\mathbf{w}} \leq$ $\|\mathbf{y}\|_{\text {block }}^{\mathbf{w}}$, given any $\mathbf{L}^{v}$ s.t. 21) is satisfied, we have $\min _{\mathcal{Q}^{v}\left(\mathbf{L}^{v}\right)}\|\overline{\mathbf{e}}\|_{\text {block }}^{\mathbf{w}} \Leftrightarrow \min _{\mathcal{Q}_{k}^{v}\left(L_{k}^{v}\right)}\left\|\overline{\mathbf{e}}_{k}\right\|_{k}(\forall k)$. The type of each component vector TICOQ $\mathcal{Q}_{k}^{v *}$ is uniquely determined by the norm $\|\cdot\|_{k}$ on $\mathbb{R}^{n_{k}}$. Furthermore, it is easy to prove that $\mathcal{Q}_{k}^{v}$ should be a lattice quantizer to minimize the worst-case error $\left\|\overline{\mathbf{e}}_{k}\right\|_{k}$. We shall discuss the two cases for $L_{2}$ norm and weighted maximum norm separately.

- $\|\cdot\|_{k}(1 \leq k \leq K)$ is $L_{2}$ norm: The covering problem asks for the thinnest covering of $\mathbb{R}^{n_{k}}$ dimensional space with overlapping spheres, i.e. minimizes covering radius (circumradius of the Voronoi cell) $\rho_{k}=\left\|\overline{\mathbf{e}}_{k}\right\|_{k}=\left(\sum_{m \in \mathcal{M}_{k}}\left|\bar{e}_{m}\right|^{2}\right)^{\frac{1}{2}}$ [24]. Therefore, each component vector TICOQ $\mathcal{Q}_{k}^{v *}$ minimizing the worst-case error is the thinnest lattice for the covering problem.

- $\|\cdot\|_{k}(1 \leq k \leq K)$ is weighted maximum norm: Given any $L_{k}^{v}$, we have $\min _{\mathcal{Q}_{k}^{v}\left(L_{k}^{v}\right)}\left\|\overline{\mathbf{e}}_{k}\right\|_{\infty}^{\mathbf{a}_{k}}=$ $\min _{\mathcal{Q}_{k}^{v}\left(L_{k}^{v}\right)} \max _{m \in \mathcal{M}_{k}} \frac{\left|\bar{e}_{m}\right|}{a_{m}}$. It can be easily shown that each face of the Voronoi cell for the weighted maximum norm is $\left(n_{k}-1\right)$ - dimensional hyperplane parallel to a coordinate axis in the $n_{k}$ dimensional space. Therefore, it is equivalent to the scalar quantization of each coordinate $x_{m}$ of the input block component $\mathbf{x}_{k}$ with different scalar quantizers, i.e. $\mathcal{Q}_{k}^{v *}=\left(\mathcal{Q}_{m}^{s *}\right)_{m \in \mathcal{M}_{k}}$.

Next, we shall show the optimal solution for the dual lattice quantizer. For $A_{n_{k}}^{*}$, the covering radius is $R_{n_{k}}=\sqrt{\frac{n_{k}\left(n_{k}+2\right)}{12\left(n_{k}+1\right)}}$, the volume of the fundamental region is $\sqrt{\frac{1}{n_{k}+1}}$. The volume of bounded region $\mathcal{X}_{k}$ is $V_{k}^{B}=\prod_{m \in \mathcal{M}_{k}}\left|\mathcal{X}_{m}\right|$. Therefore, the worst case error of the vector TICOQ with quantization rate
$L_{k}^{v}$ is given by $\left\|\overline{\mathbf{e}}_{k}\right\|_{k}=\left(\frac{V_{k}^{B}}{2^{L_{k}^{v}} \sqrt{\frac{1}{n_{k}+1}}}\right)^{\frac{1}{n_{k}}} R_{n_{k}}=$ $\left(\frac{\prod_{m \in \mathcal{M}_{k}}\left|\mathcal{X}_{m}\right|}{\sqrt{\frac{1}{n_{k}+1}}}\right)^{\frac{1}{n_{k}}} \sqrt{\frac{n_{k}\left(n_{k}+2\right)}{12\left(n_{k}+1\right)}} 2^{-\frac{L_{k}^{v}}{n_{k}}} 13$. The covering radius measured by $L_{p}$ norm $(p>2)$ can be proved to be less than $R_{n_{k}}=\sqrt{\frac{n_{k}\left(n_{k}+2\right)}{12\left(n_{k}+1\right)}}$, which is the covering radius measured by $L_{2}$ norm. Thus, the worst case error is also less than $\left\|\overline{\mathbf{e}}_{k}\right\|_{k}$ given above. Therefore, in general, we can apply $A_{n}^{*}$ quantizer for VQ case when $\|\cdot\|_{k}$ is $L_{p}$ norm $(p \geq 2)$ and consider TICOQ design for VQ cased based on $A_{n}^{*}$ quantizer.

Problem 1 for $A_{n}^{*}$ quantizer is equivalent to $\min _{\mathbf{L}^{v}}\left(\max _{k} D_{k} 2^{-\frac{L_{k}^{v}}{n_{k}}}\right)$ s.t.21). Similar to Appendix $\mathrm{D}$, the optimal solution under continuous relaxation is $\bar{L}_{k}^{v *}=n_{k}\left(\log _{2} \frac{D_{k}}{\tau}\right)^{+}$, where $\tau$ is a constant related to the LM and is chosen to satisfy $\sum_{m=1}^{n} n_{k}\left(\log _{2} \frac{D_{k}}{\tau}\right)^{+}=L$. For $n_{1}=\cdots=n_{K}$, we can use similar argument as in Appendix D to show that the rounding method in (24) is optimal integer solution to Problem 1 (VQ case).

\section{Appendix F: Proof of Lemma 5}

We try to find $L^{\prime}$ in the following three cases s.t. when $L \geq L^{\prime}$, we have $C_{m} \geq \tau(\forall m), C_{m} \geq \sum_{k=1}^{K} \tau_{k} \mathbf{I}[m \in$ $\left.\mathcal{M}_{k}\right](\forall m)$ and $D_{k} \geq \tau(\forall k)$, separately (to obtain the closed-form optimal value $\left.\left\|\overline{\mathbf{e}}^{*}\right\|_{\text {block }}^{\mathbf{w}}\right)$. Specifically, we have

- SQ $\left(\|\cdot\|_{k}\right.$ is weighted maximum norm) in Theorem 3 (same as VQ $\left(\|\cdot\|_{k}\right.$ is weighted maximum norm)): $C_{m} \geq \tau(\forall m) \Leftrightarrow \bar{L}_{m}^{s}=\left(\log _{2} \frac{C_{m}}{\tau}\right)^{+}=$ $\log _{2} \frac{C_{m}}{\tau}(\forall m)$. Since $\sum_{m=1}^{n} \log _{2} \frac{C_{m}}{\tau}=L \Rightarrow$ $\left\|\overline{\mathbf{e}}^{*}\right\|_{\text {block }}^{\mathbf{w}}=\tau=2^{\frac{1}{n} \sum_{m=1}^{n} \log _{2} C_{m}-\frac{L}{n}}=\mathcal{O}\left(2^{-\frac{L}{n}}\right)$, we have $C_{m} \geq \tau(\forall m) \Leftrightarrow \min _{m} C_{m} \geq$ $2^{\frac{1}{n} \sum_{m=1}^{n} \log _{2} C_{m}-\frac{L}{n}} \Leftrightarrow L \geq \sum_{m=1}^{n} \log _{2} C_{m}-$ $n \log _{2}\left(\min _{m} C_{m}\right) \triangleq L^{\prime}$.

- SQ (\| $\cdot \|_{k}$ is $L_{p}$ norm) in Theorem 4. $C_{m} \geq \sum_{k=1}^{K} \tau_{k} \mathbf{I}\left[m \quad \in \quad \mathcal{M}_{k}\right] \quad(\forall m) \Leftrightarrow$ $\bar{L}_{m}^{s}=\frac{1}{p} \log _{2}\left(\frac{C_{m}}{\sum_{k=1}^{K} \tau_{k} \mathbf{I}\left[m \in \mathcal{M}_{k}\right]} \vee 1\right)=$ $\frac{1}{p} \log _{2}\left(\frac{C_{m}}{\sum_{k=1}^{K} \tau_{k} \mathbf{I}\left[m \in \mathcal{M}_{k}\right]}\right) \quad(\forall m) . \quad$ Since $\sum_{m \in \mathcal{M}_{k}} C_{m} 2^{-p \bar{L}_{m}^{s}}=n_{k} \tau_{k}=\tau \Rightarrow \sum_{m=1}^{n} \bar{L}_{m}^{s}=$ $\frac{1}{p} \sum_{m=1}^{n} \log _{2} \frac{\tilde{C}_{m}}{\tau}=L \Rightarrow\left\|\overline{\mathbf{e}}^{*}\right\|_{\text {block }}^{\mathbf{w}}=$ $\tau^{\frac{1}{p}}=2^{\frac{1}{p}\left(\frac{1}{n} \sum_{m=1}^{n} \log _{2}\left(\tilde{C}_{m}\right)-\frac{p L}{n}\right)}=\mathcal{O}\left(2^{-\frac{L}{n}}\right)$. Similarly, we have $L^{\prime}=\sum_{m=1}^{n} \log _{2}\left(\tilde{C}_{m}\right)-$ $n \log _{2}\left(\min _{m} \tilde{C}_{m}\right)$.

- VQ ( $A_{n}^{*}$ quantizer) in Theorem $5, D_{k} \geq \tau(\forall k) \Leftrightarrow$ $\bar{L}_{k}^{v}=n_{k}\left(\log _{2} \frac{D_{k}}{\tau}\right)^{+}=n_{k} \log _{2} \frac{D_{k}}{\tau}(\forall k)$. Since $\sum_{k=1}^{K} n_{k} \log _{2} \frac{D_{k}}{\tau}=L \Rightarrow\left\|\overline{\mathbf{e}}^{*}\right\|_{\text {block }}^{\mathbf{w}}=\tau=$ $2^{\frac{1}{n} \sum_{k=1}^{K} n_{k} \log _{2} D_{k}-\frac{L}{n}}=\mathcal{O}\left(2^{-\frac{L}{n}}\right)$. Similarly, we have $L^{\prime}=\sum_{k=1}^{K} \log _{2} D_{k}-K \log _{2}\left(\min _{k} D_{k}\right)$.

${ }^{13}$ Note that boundary effect is ignored here. The performance loss is negligible when $L$ is large, which is easily satisfied in most of the cases we are interested in. 


\section{Appendix G: Proof of Theorem 6and Lemma 6}

First, we shall find the requirement for $L$ s.t. each subproblem (under continuous relaxation) has closedform $\left\|\tilde{\mathbf{e}}_{t}^{*}(L(t))\right\|_{\text {block }}^{\mathbf{w}}$ (to obtain the closed-form objective function of the TVCOQ master problem). By Appendix $\mathrm{F}$, to obtain closed-form $\left\|\tilde{\mathbf{e}}_{t}^{*}(L(t))\right\|_{\text {block }}^{\mathbf{w}}$, we require $L(t) \geq L^{\prime}(0 \leq t \leq \bar{T}-1)$. Under this assumption, we have $\left\|\tilde{\mathbf{e}}_{t}^{*}(L(t))\right\|_{\text {block }}^{\mathbf{w}}=\eta 2^{-\frac{L(t)}{n}}$, where

$$
\eta=\left\{\begin{array}{ll}
2^{\frac{1}{n} \sum_{m=1}^{n} \log _{2} C_{m}}, & \text { WM norm (SQ) } \\
2^{\frac{1}{p n} \sum_{m=1}^{n} \log _{2}\left(\tilde{C}_{m}\right)}, & L_{p} \text { norm (SQ) } \\
2^{\frac{1}{n} \sum_{k=1}^{K} n_{k} \log _{2} D_{k}}, & \text { dual lattice (VQ) }
\end{array} .\right.
$$

Therefore, the objective function in Problem 4 becomes $\eta \alpha^{\bar{T}-1} \sum_{t=0}^{\bar{T}-1} \alpha^{-t} 2^{-\frac{L(t)}{n}}$ and Problem 4 is equivalent to $\min _{\{L(t)\}} \sum_{t=0}^{\bar{T}-1} \alpha^{-t} 2^{-\frac{L(t)}{n}}$ s.t. (32). By continuous relaxation and standard convex optimization techniques (similar to Appendix D), we have the optimal solution (under continuous relaxation) $\bar{L}^{*}(t)=n \log _{2}\left(\frac{\alpha^{-t} \ln 2}{n \mu}\right)^{+}$. Since $L(t) \geq L^{\prime}(\forall t), \bar{L}^{*}(t)=n \log _{2}\left(\frac{\alpha^{-t} \ln 2}{n \mu}\right)$. $\sum_{t=0}^{\bar{T}-1} \bar{L}(t)=n \sum_{t=0}^{\bar{T}-1}\left(\log _{2}(\ln 2)-t \log _{2}(\alpha)-\right.$ $\left.\log _{2}(n \mu)\right)=n t \log _{2}(\ln 2)-n \frac{(\bar{T}-1) \bar{T}}{2} \log _{2}(\alpha)-$ $n t \log _{2}(n \mu)=\bar{T} L \Rightarrow \bar{L}(t)=n \frac{\bar{T}-1}{2} \log _{2}(\alpha)-$ $n l \log _{2}(\alpha)+L$. Since $L(t)$ increases with $t$, to satisfy $L(t) \geq L^{\prime}(\forall t)$, we require $L(0)=n \frac{\bar{T}-1}{2} \log _{2}(\alpha)+$ $L \geq L^{\prime} \Rightarrow L \geq L^{\prime}-n \frac{\bar{T}-1}{2} \log _{2} \alpha$. Therefore, when $L \geq L^{\prime}-n \frac{\bar{T}-1}{2} \log _{2} \alpha$, we have $\bar{L}^{*}(t)=n \log _{2}\left(\frac{\alpha^{-t} \ln 2}{n \mu}\right)$ and $\alpha^{-t}\left\|\tilde{\mathbf{e}}_{t}^{*}\left(\bar{L}^{*}(t)\right)\right\|_{\text {block }}^{\mathbf{w}}=\eta \cdot \alpha^{-t} \frac{n \mu}{\alpha^{-t} \ln 2}=\eta \cdot \frac{n \mu}{\ln 2}$. Similar to Appendix D, the rounding policy in (33) can be shown to be optimal.

Next, we shall analyze the tradeoff between convergence error and message passing overhead for $L \geq$ $L^{\prime}-n \frac{\bar{T}-1}{2} \log _{2} \alpha\left(L \in \mathbb{Z}^{+}\right)$. Since it has been shown that $\bar{L}(t)=n \frac{\bar{T}-1}{2} \log _{2}(\alpha)-n l \log _{2}(\alpha)+L$, we have $\eta \alpha^{\bar{T}-1} \sum_{t=0}^{\bar{T}-1} \alpha^{-t} 2^{-\frac{L(t)}{n}}=\eta \alpha^{\bar{T}-1} \sum_{t=0}^{\bar{T}-1} \alpha^{-t}\left(\alpha^{-\frac{\bar{T}-1}{2}}\right.$. $\left.\alpha^{t} \cdot 2^{-\frac{L}{n}}\right)=\bar{T} \alpha^{\frac{\bar{T}-1}{2}} \mathcal{O}\left(2^{-\frac{L}{n}}\right) \Rightarrow \tilde{E}_{\text {block }}^{\mathbf{w}}(\bar{T})=$ $\bar{T} \alpha^{\frac{\bar{T}-1}{2}} \mathcal{O}\left(2^{-\frac{L}{n}}\right)$.

\section{REFERENCES}

[1] D. P. Palomar and M. Chiang, "A tutorial on decomposition methods for network utillity maximization," IEEE J. Select. Areas Commun., vol. 24, no. 8, pp. 1439 - 1451, Aug. 2006.

[2] _ "Alternative decompositions and distributed algorithms for network utility maximization," in IEEE Global Telecommunications Conference (GLOBECOM), vol. 5, St. Louis, Missouri, Nov. 2005 , pp. $2563-2568$.

[3] W. Yu, G. Ginis, and J. M. Cioffi, "Distributed multiuser power control for digital subscriber lines," IEEE J. Select. Areas Commun., vol. 20, no. 5, June 2002.

[4] G. Scutari, D.P. Palomar, and S. Barbarossa, "Distributed totally asynchronous iterative waterfilling for wideband interference channel with time/frequency offset," in ICASSP, 2007, pp. 41774184.

[5] G. Scutari, D. P. Palomar, and S. Barbarossa, "Competitive design of multiuser MIMO systems based on game theory: A unified view," IEEE J. Select. Areas Commun., vol. 26, no. 7, Sept. 2008.
[6] — " "The MIMO iterative waterfilling," IEEE Trans. Signal Processing, vol. 57, no. 5, May 2009.

[7] D. P. Bertsekas and J. N. Tsitsiklis, Parallel and Distributed Computation: Numerical Methods, 1st ed. Prentice-Hall, 1989.

[8] R. P. Agarwal, M. Meehan, and D. O. Regan, Fixed Point Theory and Application. Cambrige University Press, 2001.

[9] A. Kashyap, T. Basar, and R. Srikant, "Quantized consensus," in IEEE Int. Symp. Inform. Theory (ISIT), Seattle, July 2006.

[10] T. Aysal, M. Coates, and M. G. Rabbat, "Distributed average consensus using probabilistic quantization," in Proc. IEEE Statistical Signal Processing Workshop, Madison, Aug. 2007.

[11] R. Carli, F. Fagnani, A. Speranzon, and S. Zampieri, "Communication constraints in the average consensus problem," in Automatica, vol. 44, no. 3, 2008, pp. 671-684.

[12] S. Kar and J. M.F. Moura, "Distributed consensus algorithms in sensor networks: Quantized data and random link failures," IEEE Trans. Signal Processing, vol. 58, no. 3, pp. 1383-1400, Mar. 2010.

[13] A. Nedic, A. Olshevsky, A. Ozdaglar, and J. Tsitsiklis, "On distributed averaging algorithms with quantization effects," IEEE Trans. Automat. Contr., vol. 54, no. 11, pp. 2506-2517, 2009.

[14] M. Yildiz and A. Scaglione, "Differential nested lattice encoding for consensus problems," in Proc. of IEEE Information Processing in Sensor Networks (IPSN), Apr. 2007.

[15] M. G. Rabbat and R. D. Nowak, "Quantized incremental algorithms for distributed optimization," IEEE J. Select. Areas Commun., vol. 23, no. 4, pp. 798-808, Apr. 2005.

[16] A. Nedic and A. Ozdaglar, "On the rate of convergence of distributed subgradient methods for multi-agent optimization," in Proc. of the 47th CDC Conference, 2007.

[17] A. Nedic, A. Olshevsky, A. Ozdaglar, and J. Tsitsiklis, "Distributed subgradient methods and quantization effects," in Proc. of the 47th CDC Conference, 2008, pp. 4177-4184.

[18] A. Nedic and D. P.Bertsekas, "The effect of deterministic noise in subgradient methods," in Mathematical Programming, 2009.

[19] R. M. Gray and D. L. Neuhoff, "Quantization," IEEE Trans. Inform. Theory, vol. 44, no. 6, Oct. 1998.

[20] A. Gersho and R. M. Gray, Vector Quantization and Signal Compression, 1st ed. Springer, 1992.

[21] A. Geary and D. P. Bertsekas, "Incremental subgradient methods for nondifferentiable optimization," in Proceedings of the 38th IEEE Conference on Decision and Control, vol. 1, 1999, pp. 907912.

[22] J. Huang, "Wireless resource allocation: Auctions, games and optimization," Ph.D. dissertation, Northwestern University, 2005.

[23] R. A. Horn and C. R. Johnson, Matrix Analysis. Cambrige University Press, 1986.

[24] J. H. Conway and N. J.A. Sloane, Sphere Packings, Lattices and Groups, 3rd ed. Springer, 1999.

[25] S. Boyd and L. Vandenberghe, Convex Optimization. Cambrige UK: Cambrige Univ. Press.

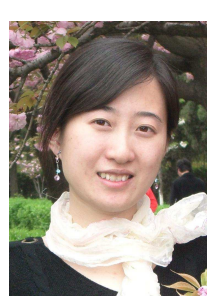

Ying Cui received B.Eng degree (first class honor) in Electronic and Information Engineering, Xian Jiaotong University, China in 2007. She is currently a Ph.D candidate in the Department of ECE, the Hong Kong University of Science and Technology (HKUST). Her current research interests include cooperative and cognitive communications, delaysensitive cross-layer scheduling as well as stochastic approximation and Markov Decision Process. 


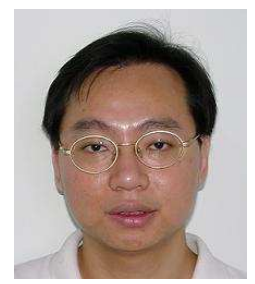

Vincent K. N. Lau obtained B.Eng (Distinction 1st Hons) from the University of Hong

Kong in 1992 and Ph.D. from Cambridge University in 1997. He was with PCCW as system engineer from 1992-1995 and Bell Labs - Lucent Technologies as member of technical staff from 1997-2003. He then joined the Department of ECE, HKUST as Associate Professor. His current research interests include the robust and delay-sensitive cross-layer scheduling, cooperative and cognitive communications as well as stochastic approximation and Markov Decision Process. 